\title{
Weak Nonlinear Double-Diffusive Magnetoconvection in a Newtonian Liquid under Temperature Modulation
}

\author{
B. S. Bhadauria and Palle Kiran \\ Department of Applied Mathematics, School for Physical Sciences, Babasaheb Bhimrao Ambedkar University, Lucknow 226 025, India \\ Correspondence should be addressed to B. S. Bhadauria; mathsbsb@yahoo.com
}

Received 22 February 2014; Accepted 15 June 2014; Published 6 July 2014

Academic Editor: Yurong Liu

Copyright (C) 2014 B. S. Bhadauria and P. Kiran. This is an open access article distributed under the Creative Commons Attribution License, which permits unrestricted use, distribution, and reproduction in any medium, provided the original work is properly cited.

\begin{abstract}
The present paper deals with a weak nonlinear theory of double-diffusive magnetoconvection in an electrically conducting Newtonian liquid, confined between two horizontal surfaces, under a constant vertical magnetic field, and subjected to imposed time-periodic thermal boundaries. The temperature of both walls is varied time periodic in this case. The disturbances are expanded in terms of power series of amplitude of convection, which is assumed to be small. Using nonautonomous Ginzburg-Landau equation, the Nusselt and Sherwood numbers obtained analytically and studied heat and mass transport in the system. Effect of various parameters on the heat and mass transport is discussed extensively. It is found that the effect of magnetic field is to stabilize the system. Further, it is also notified that the heat and mass transport can be controlled by suitably adjusting the external parameters of the system.
\end{abstract}

\section{Introduction}

Double-diffusive convection is an important fluid dynamics phenomenon that involves motions driven by two different density gradients diffusing at different rates. In doublediffusive convection, the buoyancy force is affected not only by the difference of temperatures but also by the difference of concentration of the fluid. An example of double-diffusive convection is seen in oceanography, lakes, underground water, atmospheric pollution, chemical processes, laboratory experiments, modeling of solar ponds [1], electrochemistry, magma chambers and sparks [2], Fernando and Brandt[3], formation of microstructure during the cooling of molten metals, fluid flows around shrouded heat-dissipation fins, migration of moisture through air contained in fibrous insulations, grain storage system, the dispersion of contaminants through water saturated soil, crystal $\mathrm{P}$ growth, solidification of binary mixtures, and the underground disposal of nuclear wastes. Therefore, much work has also been done on double-diffusive convection in an electrically conducted fluid layer because of its natural occurrence as mentioned above applications. Convection in planetary cores, stellar interiors, and Earth's metallic core occurs in the presence of strong magnetic field. The study of double-diffusive magnetoconvection has recently drawn the attention of astrophysicists, geophysicists, oceanographers, engineers, and a host of others $[4,5]$. The study of magnetoconvection in an electrically conducting horizontal fluid layer was motivated by astrophysical and geophysical applications; relate in some or the other way to the problems concerning the external constraints like rotation or magnetic field operative on double-diffusive systems, in particular by observation of sunspots [6].

Thompson [7] and Chandrasekhar [8] were the first to study the magnetoconvection in horizontal fluid layer. Lortz [9] was the first to study the effect of magnetic field on doublediffusive convection. His object was to clarify some of the mathematical aspects of stability criterion [10] but his analysis is silent about the detailed study of stability analysis. Stommel et al. [11] explained that the diffusion is generally a stabilizing factor in a single-component fluid. But in the case of twocomponent system it can act to release the potential energy in the component that is the heaviest at the top and make the system unstable. Gotoh and Yamada [12] studied the problem of magnetoconvection in a horizontal layer of magnetic fluid 
which is heated from below and cooled from above and found condition for onset of convection. Oreper and Szekely [13] have found that the presence of a magnetic field can suppress natural convection currents and that the strength of the magnetic field is one of the important factors in determining the quality of the crystal. Rudraiah and Shivakumara [5] investigated both linear and nonlinear theory of this problem in detail. They have shown that the magnetic field, under certain conditions, makes the system unstable. They have also investigated the effect of magnetic field on the physically preferred cell pattern. Rudraiah [14] investigated the interaction between double-diffusive convection and an externally imposed vertical magnetic field in a Boussinesq fluid. Siddheshwar and Pranesh [15] analyzed the role of magnetic field in the inhibition of natural convection driven by combined buoyancy and surface tension forces in a horizontal layer of an electrically conducting Boussinesq fluid with suspended particles confined between an upper free/adiabatic and a lower rigid/isothermal boundary is considered in $1 \mathrm{~g}$ and $\mu \mathrm{g}$ situations. Bhadauria [16] also studied the effect of magnetic field on thermal modulated convection in the case of porous medium. Siddheshwar et al. [17] performed a local nonlinear stability analysis of Rayleigh-Bénard magnetoconvection using Ginzburg-Landau equation. They showed that gravity modulation can be used to enhance or diminish the heat transport in stationary magnetoconvection.

The classical Rayleigh-Bénard convection due to bottom heating is well known and highly explored phenomenon given by Chandrasekhar [8] and Drazin and Reid [18]. Many researchers, under different physical models, have investigated thermal instability in a horizontal fluid layer with temperature modulation in the absence of doublediffusive magnetoconvection. Some of them are Venezian [19], who was the first to consider the effect of temperature modulation on thermal instability in a horizontal fluid layer. Rosenblat and Tanaka [20] studied the linear stability for a fluid in a classical geometry of Bénard by considering the temperature modulation of rigid-rigid boundaries. The first nonlinear stability problem in a horizontal fluid layer, under temperature modulation of the boundaries, was studied by Roppo et al. [21]. Bhadauria and Bhatia [22] studied the effect of temperature modulation on thermal instability by considering rigid-rigid boundaries and different types of temperature profiles. Bhadauria [23], Malashetty and Swamy [24], and Bhadauria and Kiran [25] are the related problems.

The problem of double-diffusive magnetoconvection is called thermohaline magnetoconvection when the two diffusive mechanisms are thermal and solute. From the above literature (second paragraph) it is observed that concerning linear theory numerous studies on magnetoconvection or double-diffusive magnetoconvection have been made. But, the double-diffusive magnetoconvection is still in its infant stage in nonlinear case. Liner theory gives information about onset of convection but fails at heat and mass transport. Most of the studies are made with uniform temperature gradient across the boundaries. In general there are many practical situations in which the temperature gradient is a function of both time and space. One of the external effective mechanisms to regulate (or control) the convection by maintaining

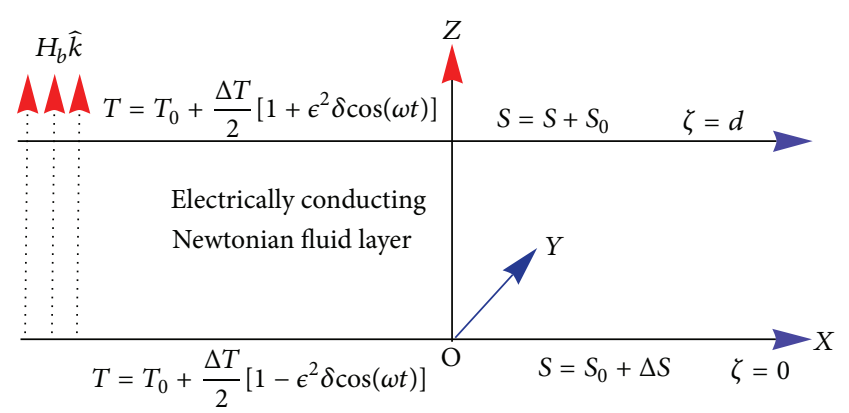

Figure 1: Physical configuration of the problem.

a nonuniform temperature gradient across the fluid layer. We also observe from the literature (third paragraph) that some work has been done under temperature modulation without magnetic field and double-diffusive convection which is essential in thermal and engineering science as mentioned in the literature. Therefore, from the above understanding we motivated to analyze a weak nonlinear thermohaline magnetoconvection under temperature modulation while deriving an amplitude of convection using Ginzburg-Landau model.

\section{Governing Equations}

We consider an electrically conducting fluid layer of depth $d$, confined between two infinite parallel horizontal planes at $z=0$ and $z=d$. A Cartesian frame of reference has been taken with the origin at the bottom, and the $z$ axis vertically upward direction of the fluid layer. The surfaces are maintained at a constant temperature gradient $\Delta T / d$ and a constant magnetic field $H_{b} \vec{k}$ is applied across the fluid layer (as shown in Figure 1). Under the Boussinesq approximation, the dimensional governing equations for the study of doublediffusive magnetoconvection in an electrically conducting fluid layer are defined as

$$
\begin{gathered}
\nabla \cdot \overrightarrow{\mathbf{q}}=0, \\
\nabla \cdot \overrightarrow{\mathbf{H}}=0 \\
\frac{\partial \overrightarrow{\mathbf{q}}}{\partial t}+(\overrightarrow{\mathbf{q}} \cdot \nabla) \overrightarrow{\mathbf{q}}=\frac{1}{\rho_{0}} \nabla p+\frac{\rho}{\rho_{0}} \overrightarrow{\mathbf{g}}-\frac{\mu}{\rho_{0}} \nabla^{2} \overrightarrow{\mathbf{q}}+\frac{\mu_{m}}{\rho_{0}} \overrightarrow{\mathbf{H}} \cdot \nabla \overrightarrow{\mathbf{H}}, \\
\gamma \frac{\partial T}{\partial t}+(\overrightarrow{\mathbf{q}} \cdot \nabla) T=\kappa_{T} \nabla^{2} T, \\
\frac{\partial S}{\partial t}+(\overrightarrow{\mathbf{q}} \cdot \nabla) S=\kappa_{S} \nabla^{2} S \\
\frac{\partial \overrightarrow{\mathbf{H}}}{\partial t}+(\overrightarrow{\mathbf{q}} \cdot \nabla) \overrightarrow{\mathbf{H}}-(\overrightarrow{\mathbf{H}} \cdot \nabla) \overrightarrow{\mathbf{q}}=v_{m} \nabla^{2} \overrightarrow{\mathbf{H}}, \\
\rho=\rho_{0}\left[1-\alpha\left(T-T_{0}\right)+\beta\left(S-S_{0}\right)\right],
\end{gathered}
$$

where $\overrightarrow{\mathbf{q}}$ is velocity $(u, v, w), \mu$ is a viscosity, $\mu_{m}$ is magnetic permeability, $\nu_{m}$ is magnetic viscosity, $\kappa_{T}$ is the thermal diffusivity, $\kappa_{S}$ is the solutal diffusivity, $T$ is temperature, 
$\alpha$ is thermal expansion coefficient, $\beta$ is solute expansion coefficient, and $\gamma$ is the ration of heat capacity. For simplicity $\gamma$ is taken to be unity in this paper, $\rho$ is density, $\overrightarrow{\mathbf{g}}=(0,0,-g)$ is gravitational acceleration, while $\rho_{0}$ is reference density, and $\overrightarrow{\mathbf{H}}$ is the magnetic field. The externally imposed thermal boundary conditions considered in this paper are given by Venezian [19]:

$$
\begin{aligned}
T & =T_{0}+\frac{\Delta T}{2}\left[1+\epsilon^{2} \delta \cos (\omega t)\right] \quad \text { at } z=0 \\
& =T_{0}-\frac{\Delta T}{2}\left[1-\epsilon^{2} \delta \cos (\omega t+\theta)\right] \quad \text { at } z=d,
\end{aligned}
$$

where $\delta$ is small amplitude of modulation, $\Delta T$ is the temperature difference across the fluid layer, $\omega$ is the modulation frequency, and $\theta$ is the phase difference. Since we are not considering cross diffusion terms, the walls of the fluid layer are assumed to be maintained at constant solute concentration as defined below:

$$
\begin{aligned}
S & =S_{0}+\Delta S \quad \text { at } z=0, \\
& =S_{0} \quad \text { at } z=d .
\end{aligned}
$$

The basic state is assumed to be quiescent and the quantities in this state are given by

$$
\begin{gathered}
\overrightarrow{\mathbf{q}}_{b}=0, \quad \rho=\rho_{b}(z, t), \quad p=p_{b}(z, t), \\
T=T_{b}(z, t), \quad S=S_{b}(z) .
\end{gathered}
$$

The basic state pressure field is not required here; however the basic temperature and solute fields are governed by the following ordinary differential equations:

$$
\frac{\partial T_{b}}{\partial t}=\kappa_{T} \frac{\partial^{2} T_{b}}{\partial z^{2}}, \quad \frac{\partial^{2} S_{b}}{\partial z^{2}}=0
$$

The solutions of (11) subjected to the boundary conditions (8)-(9) are given by

$$
\begin{gathered}
T_{b}(z, t)=T_{s}(z)+\epsilon^{2} \delta \operatorname{Re}\left[T_{1}(z, t)\right], \\
S_{b}=S_{0}+\Delta S\left(1-\frac{z}{d}\right),
\end{gathered}
$$

where $T_{s}(z)$ is the study temperature field and $T_{1}(z, t)$ is the oscillating part while Re stands for the real part. We assume finite amplitude perturbations on the basic state in the form

$$
\begin{array}{ccc}
q=q_{b}+\vec{q}^{\prime}, & \rho=\rho_{b}+\rho^{\prime}, & S=S_{b}+S^{\prime}, \\
p=p_{b}+p^{\prime}, & T=T_{b}+T^{\prime}, & \overrightarrow{\mathbf{H}}=H_{b}+\overrightarrow{\mathbf{H}}^{\prime},
\end{array}
$$

where the primes denote the quantities at the perturbations. Substituting (13) into (1)-(6) and using the basic state results, we obtain the following equations:

$$
\begin{gathered}
\nabla \cdot \overrightarrow{\mathbf{q}}^{\prime}=0 \\
\nabla \cdot \overrightarrow{\mathbf{H}}^{\prime}=0 \\
\frac{\partial \overrightarrow{\mathbf{q}}^{\prime}}{\partial t}+\left(\overrightarrow{\mathbf{q}}^{\prime} \cdot \nabla\right) \overrightarrow{\mathbf{q}}-\frac{\mu_{m}}{\rho_{0}}\left(\overrightarrow{\mathbf{H}}^{\prime} \cdot \nabla\right) \overrightarrow{\mathbf{H}}^{\prime} \\
=\frac{1}{\rho_{0}} \nabla p^{\prime}-\frac{\rho^{\prime}}{\rho_{0}} \mathbf{g k}+\nu \nabla^{2} \overrightarrow{\mathbf{q}}^{\prime}+\frac{\mu_{m}}{\rho_{0}} H_{b} \frac{\partial \overrightarrow{\mathbf{H}}^{\prime}}{\partial z}, \\
\frac{\partial T^{\prime}}{\partial t}+\left(\overrightarrow{\mathbf{q}}^{\prime} \cdot \nabla\right) T^{\prime}+\overrightarrow{\mathbf{w}}^{\prime} \frac{\partial T_{b}}{\partial z}=\kappa_{T} \nabla^{2} T^{\prime}, \\
\frac{\partial S^{\prime}}{\partial t}+\left(\overrightarrow{\mathbf{q}}^{\prime} \cdot \nabla\right) S^{\prime}+\overrightarrow{\mathbf{w}}^{\prime} \frac{\partial S_{b}}{\partial z}=\kappa_{S} \nabla^{2} S^{\prime}, \\
\frac{\partial \overrightarrow{\mathbf{H}}^{\prime}}{\partial t}+\left(\overrightarrow{\mathbf{q}}^{\prime} \cdot \nabla\right) \overrightarrow{\mathbf{H}}^{\prime}-\left(\overrightarrow{\mathbf{H}}^{\prime} \cdot \nabla\right) \overrightarrow{\mathbf{q}}^{\prime}-H_{b} \frac{\partial \overrightarrow{\mathbf{w}}^{\prime}}{\partial z}=\nu_{m} \nabla^{2} \overrightarrow{\mathbf{H}}^{\prime}, \\
\rho^{\prime}=-\rho_{0}\left(\alpha T^{\prime}-\beta S^{\prime}\right) .
\end{gathered}
$$

Further we consider only two-dimensional disturbances in our study and hence the stream function $\psi$ and magnetic potential $\Phi$ are introduced as $\left(\overrightarrow{\mathbf{u}}^{\prime}, \overrightarrow{\mathbf{w}}^{\prime}\right)=(\partial \psi / \partial z,-\partial \psi / \partial x)$ and $\left(\overrightarrow{\mathbf{H}}_{x}^{\prime}, \overrightarrow{\mathbf{H}}_{z}^{\prime}\right)=(\partial \Phi / \partial z,-\partial \Phi / \partial x)$. By eliminating density and pressure terms from (14) and nondimensionalizing using the following transformations: $\left(x^{\prime}, y^{\prime}, z^{\prime}\right)=d\left(x^{*}, y^{*}, z^{*}\right)$, $\psi=\kappa_{T} \psi^{*}, t=\left(d^{2} / \kappa_{T}\right) t^{*}, \overrightarrow{\mathbf{q}}^{\prime}=\left(\kappa_{T} / d\right) \overrightarrow{\mathbf{q}}^{*}, T^{\prime}=\Delta T T^{*}, S^{\prime}=$ $\Delta S S^{*}, \Phi=d H_{b} \Phi^{*}$, and $\omega=\left(\kappa_{T} / d^{2}\right) \omega^{*}$, finally dropping the asterisk for simplicity, we get the nondimensionalized governing system of equations:

$$
\begin{aligned}
& -\nabla^{4} \psi+\operatorname{Ra}_{T} \frac{\partial T}{\partial x}-\operatorname{Ras} \frac{\partial S}{\partial x}-Q \operatorname{Pm} \frac{\partial}{\partial z}\left(\nabla^{2} \Phi\right) \\
& =-\frac{1}{\operatorname{Pr}} \frac{\partial}{\partial t}\left(\nabla^{2} \psi\right)+\frac{1}{\operatorname{Pr}} \frac{\partial\left(\psi, \nabla^{2} \psi\right)}{\partial(x, z)}-Q \operatorname{Pm} \frac{\partial\left(\Phi, \nabla^{2} \Phi\right)}{\partial(x, z)},
\end{aligned}
$$

$$
-\frac{\partial \psi}{\partial x} \frac{\partial T_{b}}{\partial z}-\nabla^{2} T=-\frac{\partial T}{\partial t}+\epsilon^{2} \delta f_{2}(z, t) \frac{\partial \psi}{\partial x}+\frac{\partial(\psi, T)}{\partial(x, z)}
$$

$$
\begin{gathered}
-\frac{\partial \psi}{\partial x} \frac{\partial S_{b}}{\partial z}-\frac{1}{\mathrm{Le}} \nabla^{2} S=-\frac{\partial S}{\partial t}+\frac{\partial(\psi, S)}{\partial(x, z)} \\
-\frac{\partial \psi}{\partial z}-\operatorname{Pm} \nabla^{2} \Phi=-\frac{\partial \Phi}{\partial t}+\frac{\partial(\psi, \Phi)}{\partial(x, z)}
\end{gathered}
$$

Here the nondimensionalizing parameters in the above equations are $\mathrm{Pm}=v_{m} / \kappa_{T}$ magnetic Prandtl number, $\mathrm{Pr}=$ $\nu / \kappa_{T}$ Prandtl number, Le $=\kappa_{T} / \kappa_{S}$ is Lewis number, $\mathrm{Ra}_{T}=$ $\alpha g \Delta T d^{3} / \nu \kappa_{T}$ is thermal Rayleigh number, Ras $=\beta g \Delta S d^{3} / \nu \kappa_{T}$ is Solutal Rayleigh number, and $Q=\mu_{m} H_{b}^{2} d^{2} / \rho_{0} \nu \nu_{m}$ is Chandrasekhar number. In (16), one can observe that 
the basic state solution influences the stability problem through the factor $\partial T_{b} / \partial z$, which is given by

$$
\frac{\partial T_{b}}{\partial z}=-1+\epsilon^{2} \delta\left[f_{2}(z, t)\right]
$$

where

$$
f_{2}(z, t)=\operatorname{Re}\left[f(z) e^{-i \omega t}\right]
$$

$f(z)=\left[A(\lambda) e^{\lambda z}+A(-\lambda) e^{-\lambda z}\right], A(\lambda)=(\lambda / 2)\left(\left(e^{-i \theta}-e^{-\lambda}\right) /\left(e^{\lambda}-\right.\right.$ $\left.e^{-\lambda}\right)$ ), and $\lambda=(1-i) \sqrt{\omega / 2}$. We assume small variations of time and rescaling it as $\tau=\epsilon^{2} t$, to study the stationary convection of the system, we write the nonlinear Equations (15)-(18) in the matrix form as given below:

$$
\begin{gathered}
{\left[\begin{array}{cccc}
-\nabla^{4} & \operatorname{Ra}_{T} \frac{\partial}{\partial x} & -\operatorname{Ras} \frac{\partial}{\partial x} & -Q P m \frac{\partial}{\partial z} \nabla^{2} \\
\frac{\partial}{\partial x} & -\nabla^{2} & 0 & 0 \\
\frac{\partial}{\partial x} & 0 & -\frac{1}{\operatorname{Le}} \nabla^{2} & 0 \\
-\frac{\partial}{\partial z} & 0 & 0 & -\operatorname{Pm} \nabla^{2}
\end{array}\right]\left[\begin{array}{c}
\psi \\
T \\
S \\
\Phi
\end{array}\right]} \\
=\left[\begin{array}{c}
\frac{-1}{\operatorname{Pr}} \frac{\partial}{\partial t}\left(\nabla^{2} \psi\right)+\frac{1}{\operatorname{Pr}} \frac{\partial\left(\psi, \nabla^{2} \psi\right)}{\partial(x, z)}-Q \operatorname{Pm} \frac{\partial\left(\Phi, \nabla^{2} \Phi\right)}{\partial(x, z)} \\
-\frac{\partial T}{\partial t}+\epsilon^{2} \delta f_{2}(z, t) \frac{\partial \psi}{\partial x}+\frac{\partial(\psi, T)}{\partial(x, z)} \\
-\frac{\partial S}{\partial t}+\frac{\partial(\psi, S)}{\partial(x, z)} \\
-\frac{\partial \Phi}{\partial t}+\frac{\partial(\psi, \Phi)}{\partial(x, z)}
\end{array}\right]
\end{gathered}
$$

The considered stress free and isothermal boundary conditions to solve the above system (21) are

$$
\begin{aligned}
& \psi=0=\nabla^{2} \psi, \quad \Phi=D \Phi=0 \quad \text { at } z=0, \\
& \psi=0=\nabla^{2} \psi, \quad \Phi=D \Phi=0 \quad \text { at } z=1,
\end{aligned}
$$

where $D=\partial / \partial z$.

\section{Finite Amplitude Equation, Heat and Mass Transport}

We now introduce the following asymptotic expansions in (21):

$$
\begin{gathered}
\mathrm{Ra}_{T}=R_{0 c}+\epsilon^{2} R_{2}+\epsilon^{4} R_{4}+\cdots, \\
\psi=\epsilon \psi_{1}+\epsilon^{2} \psi_{2}+\epsilon^{3} \psi_{3}+\cdots, \\
T=\epsilon T_{1}+\epsilon^{2} T_{2}+\epsilon^{3} T_{3}+\cdots, \\
S=\epsilon S_{1}+\epsilon^{2} S_{2}+\epsilon^{3} S_{3}+\cdots, \\
\Phi=\epsilon \Phi_{1}+\epsilon^{2} \Phi_{2}+\epsilon^{3} \Phi_{3}+\cdots,
\end{gathered}
$$

where $R_{0 c}$ is the critical value of the Rayleigh number at which the onset of convection takes place in the absence of temperature modulation. Now we solve the system for different orders of $\epsilon$.

At the lowest order, we have

$$
\left[\begin{array}{cccc}
-\nabla^{4} & R_{0 c} \frac{\partial}{\partial x} & -\operatorname{Ras} \frac{\partial}{\partial x} & -\mathrm{QPm} \frac{\partial}{\partial z}\left(\nabla^{2}\right) \\
\frac{\partial}{\partial x} & -\nabla^{2} & 0 & 0 \\
\frac{\partial}{\partial x} & 0 & -\frac{1}{\mathrm{Le}} \nabla^{2} & 0 \\
-\frac{\partial}{\partial z} & 0 & 0 & -\mathrm{Pm} \nabla^{2}
\end{array}\right]\left[\begin{array}{c}
\psi_{1} \\
T_{1} \\
S_{1} \\
\Phi_{1}
\end{array}\right]
$$

$$
=\left[\begin{array}{l}
0 \\
0 \\
0 \\
0
\end{array}\right] \text {. }
$$

The solutions of the lowest order of the system subject to the boundary conditions equation (22) are

$$
\begin{gathered}
\psi_{1}=A(\tau) \sin \left(k_{c} x\right) \sin (\pi z), \\
T_{1}=-\frac{k_{c}}{\delta^{2}} A(\tau) \cos \left(k_{c} x\right) \sin (\pi z), \\
S_{1}=-\frac{k_{c}}{\delta^{2}} \operatorname{Le} A(\tau) \cos \left(k_{c} x\right) \sin (\pi z), \\
\Phi_{1}=\frac{\pi}{\operatorname{Pm} \delta^{2}} A(\tau) \sin \left(k_{c} x\right) \cos (\pi z),
\end{gathered}
$$

where $\delta^{2}=k_{c}^{2}+\pi^{2}$. The critical value of the Rayleigh number for the onset of magnetoconvection in the absence of temperature modulation is

$$
R_{0 c}=\frac{\delta^{2}\left(\delta^{4}+Q \pi^{2}\right)+\operatorname{Ras} k_{c}^{2} \mathrm{Le}}{k_{c}^{2}},
$$

which is the same as Siddheshwar et al. [17] when Ras $=0$ and we obtain classical results of Chandrasekhar [8] for without magnetic field and single component fluid layer. 
At the second order, we have

$$
\left[\begin{array}{cccc}
-\nabla^{4} & R_{0 c} \frac{\partial}{\partial x} & -\operatorname{Ras} \frac{\partial}{\partial x} & -Q \operatorname{Pm} \frac{\partial}{\partial z} \nabla^{2} \\
\frac{\partial}{\partial x} & -\nabla^{2} & 0 & 0 \\
\frac{\partial}{\partial x} & 0 & -\frac{1}{\mathrm{Le}} \nabla^{2} & 0 \\
-\frac{\partial}{\partial z} & 0 & 0 & -\mathrm{Pm} \nabla^{2}
\end{array}\right]\left[\begin{array}{c}
\psi_{2} \\
T_{2} \\
S_{2} \\
\Phi_{2}
\end{array}\right]
$$$$
=\left[\begin{array}{l}
R_{21} \\
R_{22} \\
R_{23} \\
R_{24}
\end{array}\right],
$$

$$
\begin{gathered}
R_{21}=0 \\
R_{22}=\frac{\partial \psi_{1}}{\partial x} \frac{\partial T_{1}}{\partial z}-\frac{\partial \psi_{1}}{\partial z} \frac{\partial T_{1}}{\partial x} \\
R_{23}=\frac{\partial \psi_{1}}{\partial x} \frac{\partial S_{1}}{\partial z}-\frac{\partial \psi_{1}}{\partial z} \frac{\partial S_{1}}{\partial x} \\
R_{24}=\frac{\partial \psi_{1}}{\partial x} \frac{\partial \Phi_{1}}{\partial z}-\frac{\partial \psi_{1}}{\partial z} \frac{\partial \Phi_{1}}{\partial x}
\end{gathered}
$$

The second order solutions subjected to the boundary conditions Equation (22) are obtained as follows:

$$
\begin{gathered}
\psi_{2}=0, \\
T_{2}=-\frac{k_{c}^{2}}{8 \pi \delta^{2}} A^{2}(\tau) \sin (2 \pi z), \\
S_{2}=-\frac{k_{c}^{2} \mathrm{Le}^{2}}{8 \pi \delta^{2}} A^{2}(\tau) \sin (2 \pi z), \\
\Phi_{2}=-\frac{\pi^{2}}{8 k_{c} \mathrm{Pm}^{2} \delta^{2}} A^{2}(\tau) \sin \left(2 k_{c} x\right) .
\end{gathered}
$$

The horizontally averaged Nusselt Nu and Sherwood Sh numbers for the stationary double-diffusive magnetoconvection (the mode considered in this problem) are given by

$$
\begin{aligned}
& \mathrm{Nu}(\tau)=1+\frac{\left[\left(k_{c} / 2 \pi\right) \int_{0}^{2 \pi / k_{c}}\left(\partial T_{2} / \partial z\right) d x\right]_{z=0}}{\left[\left(k_{c} / 2 \pi\right) \int_{0}^{2 \pi / k_{c}}\left(\partial T_{b} / \partial z d x\right]_{z=0}\right.}, \\
& \operatorname{Sh}(\tau)=1+\frac{\left[\left(k_{c} / 2 \pi\right) \int_{0}^{2 \pi / k_{c}}\left(\partial S_{2} / \partial z\right) d x\right]_{z=0}}{\left[\left(k_{c} / 2 \pi\right) \int_{0}^{2 \pi / k_{c}}\left(d S_{b} / d z\right) d x\right]_{z=0}} .
\end{aligned}
$$

One must note here that $f_{2}(z, \tau)$ is effective at $O\left(\epsilon^{2}\right)$ and affects $\mathrm{Nu}(\tau)$ and $\operatorname{Sh}(\tau)$ through $A(\tau)$ can be seen later. Therefore, substituting $T_{2}, T_{b}, S_{2}$, respectively, into (29) and simplifying we obtain

$$
\begin{gathered}
\mathrm{Nu}(\tau)=1+\frac{k_{c}^{2}}{4 \delta^{2}} A^{2}(\tau), \\
\mathrm{Sh}(\tau)=1+\frac{k_{c}^{2} \mathrm{Le}^{2}}{4 \delta^{2}} A^{2}(\tau) .
\end{gathered}
$$

At the third order, we have

$$
\begin{gathered}
{\left[\begin{array}{cccc}
-\nabla^{4} & R_{0 c} \frac{\partial}{\partial x} & -\operatorname{Ras} \frac{\partial}{\partial x} & -Q P m \frac{\partial}{\partial z} \nabla^{2} \\
\frac{\partial}{\partial x} & -\nabla^{2} & 0 & 0 \\
\frac{\partial}{\partial x} & 0 & -\frac{1}{\operatorname{Le}} \nabla^{2} & 0 \\
-\frac{\partial}{\partial z} & 0 & 0 & -\mathrm{Pm} \nabla^{2}
\end{array}\right]\left[\begin{array}{c}
\psi_{3} \\
T_{3} \\
S_{3} \\
\Phi_{3}
\end{array}\right]} \\
=\left[\begin{array}{l}
R_{31} \\
R_{32} \\
R_{33} \\
R_{34}
\end{array}\right],
\end{gathered}
$$

where

$$
\begin{gathered}
R_{31}=-\frac{1}{\operatorname{Pr}} \frac{\partial}{\partial \tau}\left(\nabla^{2} \psi_{1}\right)-R_{2} \frac{\partial T_{1}}{\partial x}-\operatorname{Ras} \frac{\partial S_{1}}{\partial x} \\
-Q \operatorname{Pm}\left(\frac{\partial \Phi_{1}}{\partial z} \frac{\partial}{\partial x}\left(\nabla^{2} \Phi_{2}\right)-\frac{\partial \Phi_{2}}{\partial x} \frac{\partial}{\partial z}\left(\nabla^{2} \Phi_{1}\right)\right) \\
R_{32}=-\frac{\partial T_{1}}{\partial \tau}+\delta f_{2}(z, \tau) \frac{\partial \psi_{1}}{\partial x}+\frac{\partial \psi_{1}}{\partial x} \frac{\partial T_{2}}{\partial z} \\
R_{33}=-\frac{\partial S_{1}}{\partial \tau}+\frac{\partial \psi_{1}}{\partial x} \frac{\partial S_{2}}{\partial z} \\
R_{34}=-\frac{\partial \Phi_{1}}{\partial \tau}-\frac{\partial \psi_{1}}{\partial z} \frac{\partial \Phi_{2}}{\partial x}
\end{gathered}
$$

Substituting $\psi_{1}, T_{1}$, and $T_{2}$ into (32), we obtain the expressions for $R_{31}, R_{32}, R_{33}$, and $R_{34}$ easily. For the existence of third order solution of the system we apply the solvability condition which leads to arrive at the nonautonomous GinzburgLandau equation for stationary mode of convection with time-periodic coefficients in the form

$$
A_{1} A^{\prime}(\tau)-A_{2} A(\tau)+A_{3} A(\tau)^{3}=0,
$$

where

$$
\begin{gathered}
A_{1}=\left[\frac{\delta^{2}}{\mathrm{Pr}}+\frac{R_{0 c} k_{c}^{2}}{\delta^{4}}-\frac{\mathrm{Rask}_{c}^{2} \mathrm{Le}^{2}}{\delta^{4}}-\frac{\mathrm{Q} \pi^{2}}{\mathrm{Pm} \delta^{2}}\right], \\
A_{2}=\left[\frac{R_{2} k_{c}^{2}}{\delta^{2}}-\frac{2 R_{0 c} k_{c}^{2}}{\delta^{2}} \delta I_{1}\right], \\
A_{3}=\left[\frac{R_{0 c} k_{c}^{4}}{8 \delta^{4}}+\frac{Q \pi^{4} k_{c}^{2}}{2 \mathrm{Pm}^{2} \delta^{4}}-\frac{Q \pi^{4}}{4 \mathrm{Pm}^{2} \delta^{2}}-\frac{\mathrm{Ras}_{c}^{4} \mathrm{Le}^{3}}{8 \delta^{4}}\right], \\
I_{1}=\int_{0}^{1} f_{2}(z, \tau) \sin ^{2}(\pi z) d z .
\end{gathered}
$$

The Ginzburg-Landau equations given in (33) are Bernoulli equation and obtaining its analytical solution is difficult, due to its nonautonomous nature. So that, it has been solved numerically using the in-built function NDSolve of Mathematica 8, subjected to the initial condition $A(0)=a_{0}$, where $a_{0}$ is the chosen initial amplitude of convection. In our calculations we may use $R_{2}=R_{0 c}$, to keep the parameters to the minimum. 


\section{Results and Discussion}

External regulation of thermal instability is important to study the double-diffusive convection in a fluid layer. The objective of this paper is to consider two such candidates, namely, vertical magnetic field and temperature modulation for either enhancing or inhibiting convective heat and mass transport as is required by a real application. The present paper deals with double-diffusive magnetoconvection under temperature modulation by using Ginzburg-Landau equation. It is necessary to consider a nonlinear theory to analyze heat and mass transfer which is not possible by the linear theory. We consider the direct mode $\left(\kappa_{S} / \kappa_{T}<\right.$ 1 otherwise Hopf mode) in which the salt and heat make opposing contributions $\left(\kappa_{T} \neq \kappa_{S}\right)$. We also consider the effect of temperature modulation to be of order $O\left(\epsilon^{2}\right)$; this leads to small amplitude of modulation. Such an assumption will help us in obtaining the amplitude equation of convection in a rather simple and elegant manner and is much easier to obtain than in the case of the Lorenz model. We give the following features of the problem before our results:

(1) the need for nonlinear stability analysis,

(2) the relation of the problem to a real application,

(3) the selection of all dimensionless parameters utilized in computations.

We consider the following three types of temperature modulation on the boundaries of the system:

(1) in-phase modulation [IPM] $(\theta=0)$,

(2) out-of-phase modulation [OPM] $(\theta=\pi)$,

(3) lower boundary modulation [LBMO] $(\theta=-i \infty)$.

The parameters of the system are $Q, \operatorname{Pr}$, Le, Pm, Ras, $\theta, \delta, \omega$; these parameters influence the convective heat and mass transfer. The first five parameters are related to the fluid layer, and the last three parameters concern the external mechanisms of controlling convection. The effect of temperature modulation is represented by amplitude $\delta$, which lies around 0.3 due to the assumption. The effect of electrical conductivity and magnetic field comes through $\mathrm{Pm}, \mathrm{Q}$. There is the property of the fluid coming into picture as well as through Prandtl number Pr. Further, the modulation of the boundary temperature is assumed to be of low frequency. At low range of frequencies the effect of frequency on onset of convection as well as on heat and mass transport is minimal. This assumption is required in order to ensure that the system does not pick up oscillatory convective mode at onset due to modulation in a situation that is conductive otherwise to stationary mode. It is important at this stage to consider the effect of $Q, \operatorname{Pr}, \mathrm{Le}, \operatorname{Pm}, \operatorname{Ras}, \theta, \delta, \omega$ on the onset of convection. The heat and mass transfer of the problem are quantified by the Nusselt and Sherwood numbers which are given in (30). Figures 2-7 show the individual effect of each nondimensional parameter on heat and mass transfer.

(1) Figures 2(a)-7(a) show that the effect of Chandrasekhar number $Q$ which is ratio of Lorentz force to viscous force is to delay the onset of convection, hence heat and mass transfer. The Nu and Sh start with one and for small values of time $\tau$ increase and become constant for large values of time $\tau$ in the case of [IPM] given in Figures 2(a) and 5(a). In the case of [OPM, LBMO] the effect of $Q$ shows oscillatory behaviour and increment in it decreases the magnitude of both $\mathrm{Nu}$ and Sh. Hence $Q$ has stabilizing effect in all the three types of modulations given in Figures 3(a), 4(a), 6(a), and 7(a), so that heat and mass transfer decrease with $Q$.

(2) The effect of Prandtl number Pr is to advance the convection and hence heat and mass transfer, which has similar behaviour of (1), given in Figures 2(b)-7(b).

(3) The effect of Lewis Le and Magnetic Prandtl Pm numbers is to advance the convection and hence heat and mass transfer. Hence both Le and Pm have destabilizing effect of the system given in Figures 2(c)-7(c) and 2(d)-7(d) and have similar behaviour of (1); these results are earlier obtained by Siddheshwar et al. [17].

(4) The effect of solutal Rayleigh number Ras is to increase $\mathrm{Nu}$ and $\mathrm{Sh}$ so that heat and mass transfer. Hence it has destabilizing effect in all the three types of modulations which is given by the Figures 2(e)-7(e) and has similar behaviour of (1). Though the presence of a stabilizing gradient of solute will prevent the onset of convection, the strong finite-amplitude motions, which exist for large Rayleigh numbers, tend to mix the solute and redistribute it so that the interior layers of the fluid are more neutrally stratified. As a consequence, the inhibiting effect of the solute gradient is greatly reduced and hence fluid will convect more and more heat and mass when Ras is increased.

(5) In the case of [IPM] we observe no effect of amplitude $\delta$ and frequency $\omega$ of modulation which is given by the Figures 2(f) and 5(f). But in the case of [OPM-LBMO] the increment in $\delta$ leads to increment in magnitude of $\mathrm{Nu}$ and Sh hence heat and mass transfer given in Figures 3(f), 4(f), $6(\mathrm{f})$, and $7(\mathrm{f})$; the increment in $\omega$ shortens the wavelength and decreases in magnitude of $\mathrm{Nu}, \mathrm{Sh}$ and hence heat and mass transfer given in Figures 3(g), 4(g), 6(g), and 7(g) which are the results obtained by Venezian [19], Siddheshwar et al. [17], and Bhadauria and Kiran [25].

(6) From Figures $2(\mathrm{~g})$ and $5(\mathrm{~g})$ we observe that $Q$ has strongly stabilizing effect. $[\mathrm{Nu} / \mathrm{Sh}]_{\mathrm{Q}=0}>[\mathrm{Nu} / \mathrm{Sh}]_{\mathrm{Q} \neq 0}$.

(7) The comparison of three types of temperature modulations is given in Figures $4(\mathrm{~h})$ and $7(\mathrm{~h}):[\mathrm{Nu} / \mathrm{Sh}]^{\mathrm{IPM}}<$ $[\mathrm{Nu} / \mathrm{Sh}]^{\mathrm{LBMO}}<[\mathrm{Nu} / \mathrm{Sh}]^{\mathrm{OPM}}$.

(8) The results of this work can be summarized as follows from Figures 2-7.

(1) $[\mathrm{Nu} / \mathrm{Sh}]_{\mathrm{Q}=25}<[\mathrm{Nu} / \mathrm{Sh}]_{\mathrm{Q}=15}<[\mathrm{Nu} / \mathrm{Sh}]_{\mathrm{Q}=10}$, Figures 2(a) $-7(\mathrm{a})$.

(2) $[\mathrm{Nu} / \mathrm{Sh}]_{\operatorname{Pr}=0.5}<[\mathrm{Nu} / \mathrm{Sh}]_{\operatorname{Pr}=1.0}<[\mathrm{Nu} / \mathrm{Sh}]_{\operatorname{Pr}=1.5}$, Figures 2(b)-7(b).

(3) $[\mathrm{Nu}]_{\mathrm{Le}=1.2}<[\mathrm{Nu}]_{\mathrm{Le}=4.2}<[\mathrm{Nu}]_{\mathrm{Le}=6.2}$, Figures 2(c), $3(\mathrm{c})$, and 4(c).

(4) $[\mathrm{Sh}]_{\mathrm{Le}=1.2}<[\mathrm{Sh}]_{\mathrm{Le}=1.4}<[\mathrm{Sh}]_{\mathrm{Le}=1.6}$, Figures 5(c), 6(c), and $7(\mathrm{c})$.

(5) $[\mathrm{Nu} / \mathrm{Sh}]_{\mathrm{Pm}=1.2}<[\mathrm{Nu} / \mathrm{Sh}]_{\mathrm{Pm}=1.4}<[\mathrm{Nu} / \mathrm{Sh}]_{\mathrm{Pm}=1.6}$, Figures 2(d) $-7(\mathrm{~d})$. 


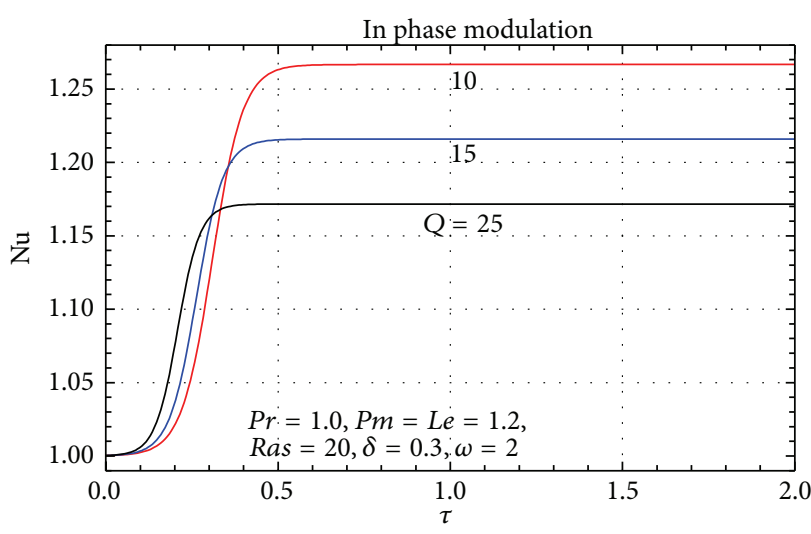

(a)

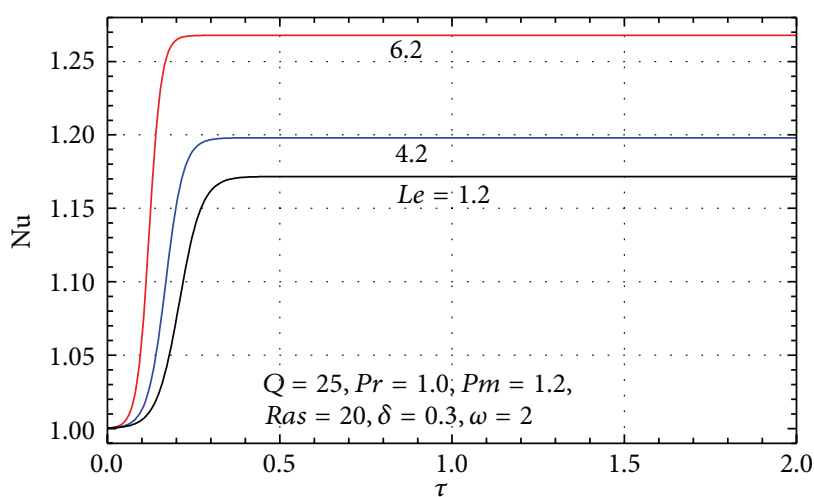

(c)

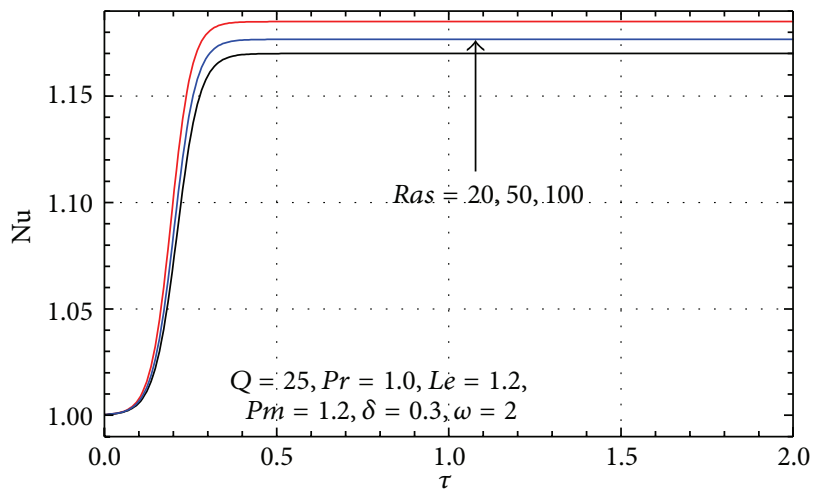

(e)

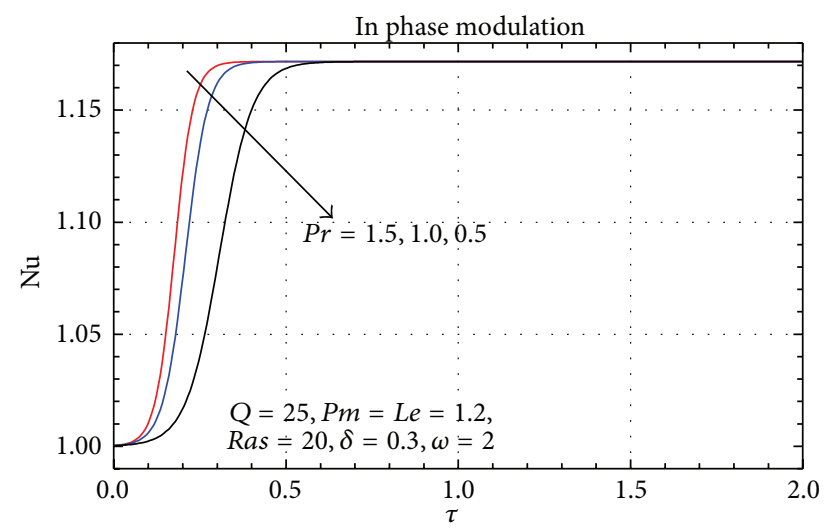

(b)

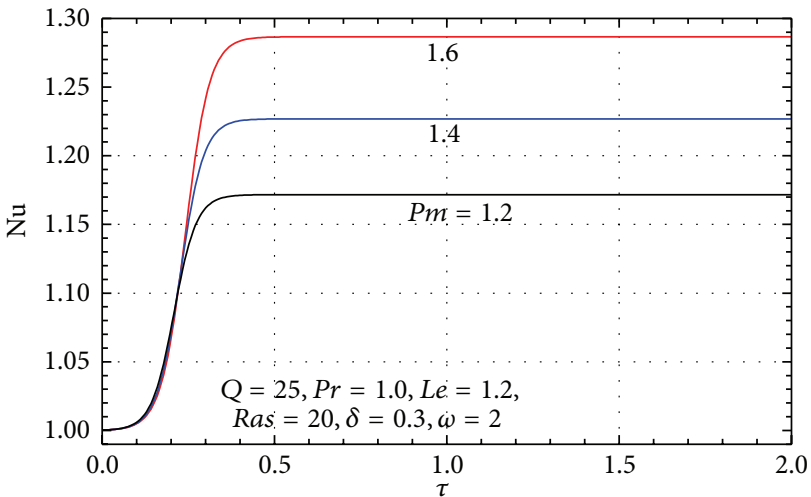

(d)

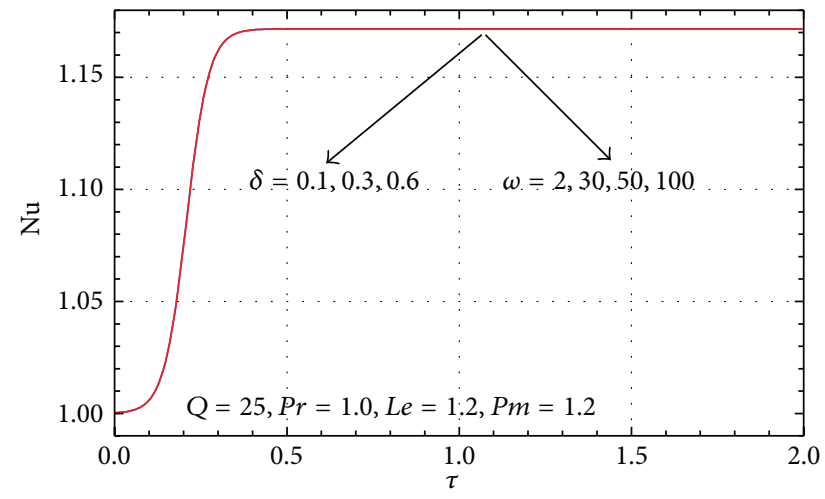

(f)

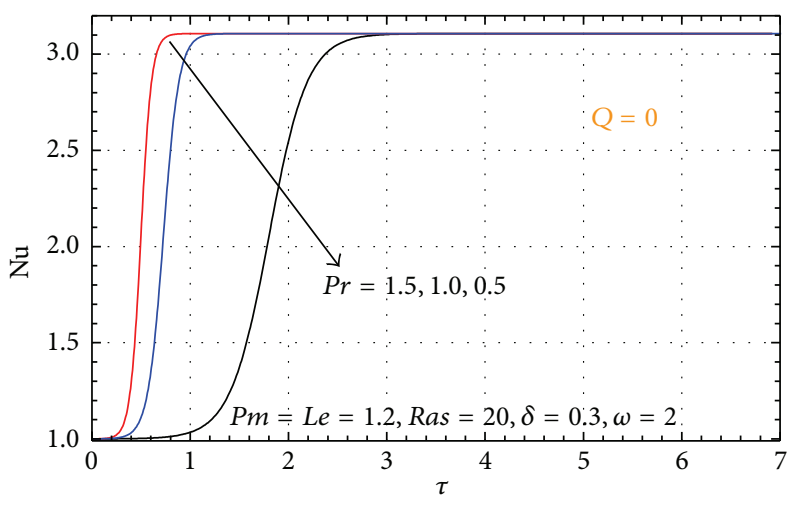

(g)

Figure 2: Nu versus $\tau$ (a) $Q$ (b) $\operatorname{Pr}(\mathrm{c}) \operatorname{Le}(\mathrm{d}) \operatorname{Pm}(\mathrm{e}) \operatorname{Ras}(\mathrm{f}) \delta, \omega$. 


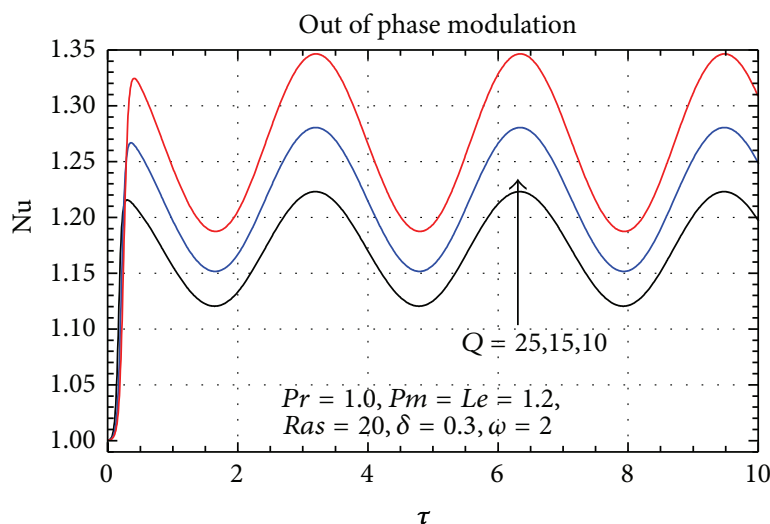

(a)

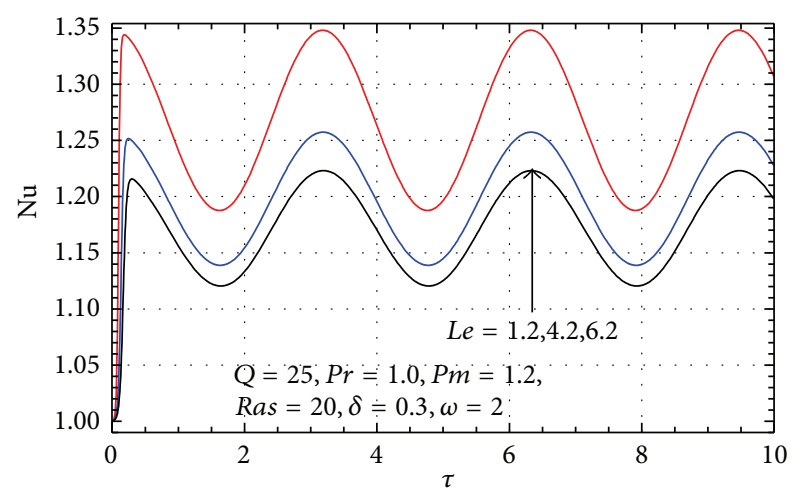

(c)

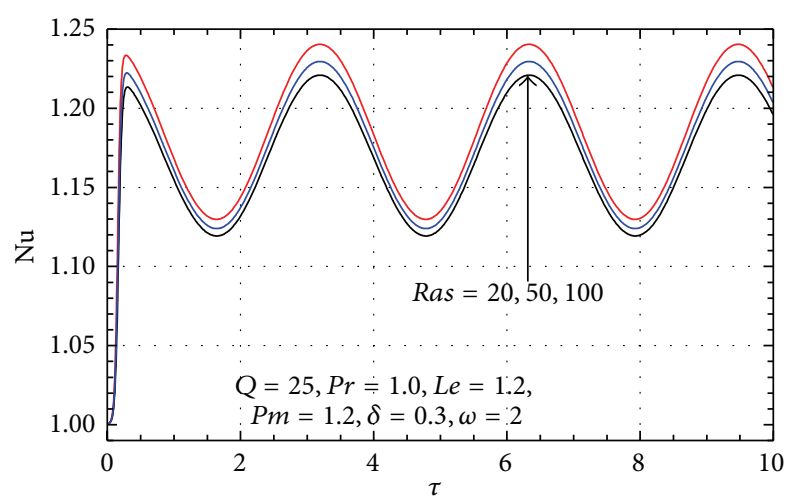

(e)

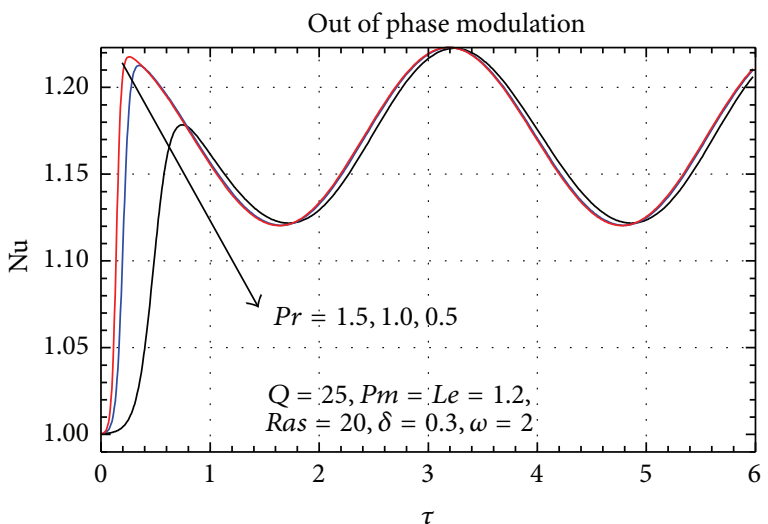

(b)

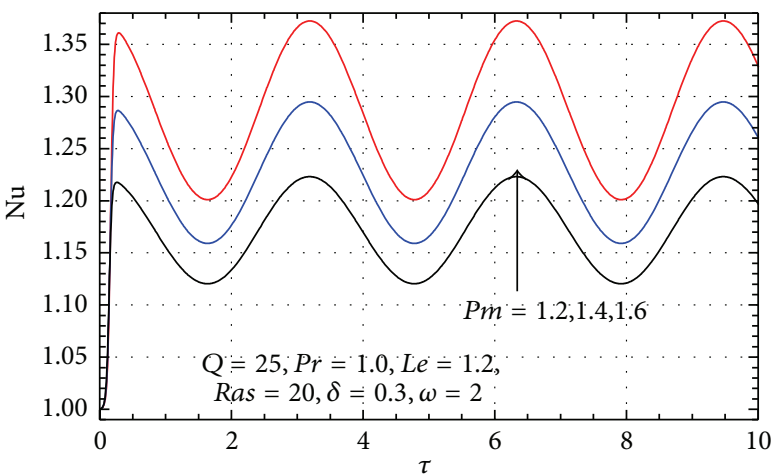

(d)

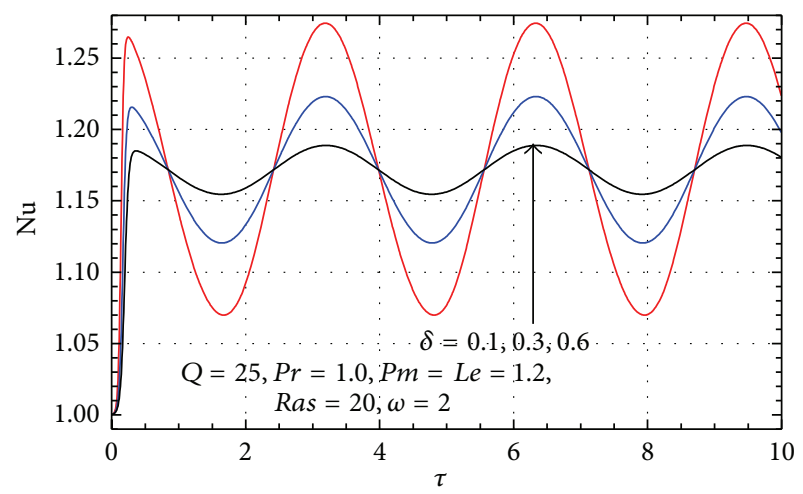

(f)

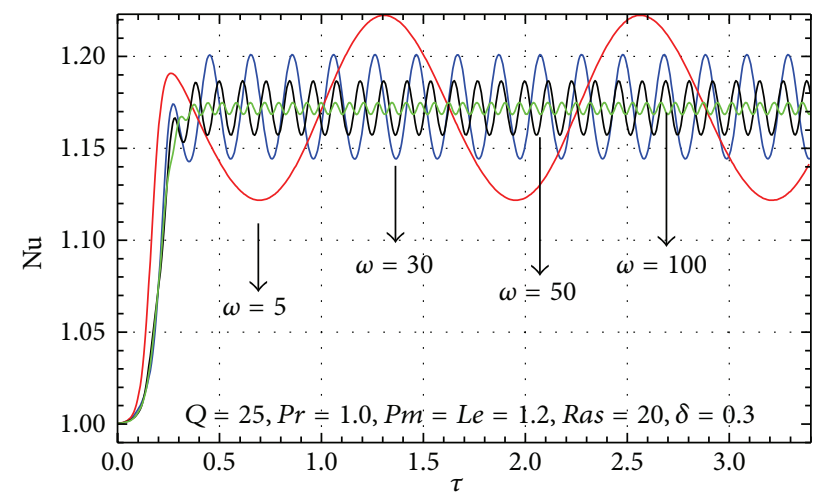

(g)

Figure 3: Nu versus $\tau$ (a) $Q$ (b) $\operatorname{Pr}(\mathrm{c}) \operatorname{Le}(\mathrm{d}) \operatorname{Pm}(\mathrm{e}) \operatorname{Ras}(\mathrm{f}) \delta$ (g) $\omega$. 


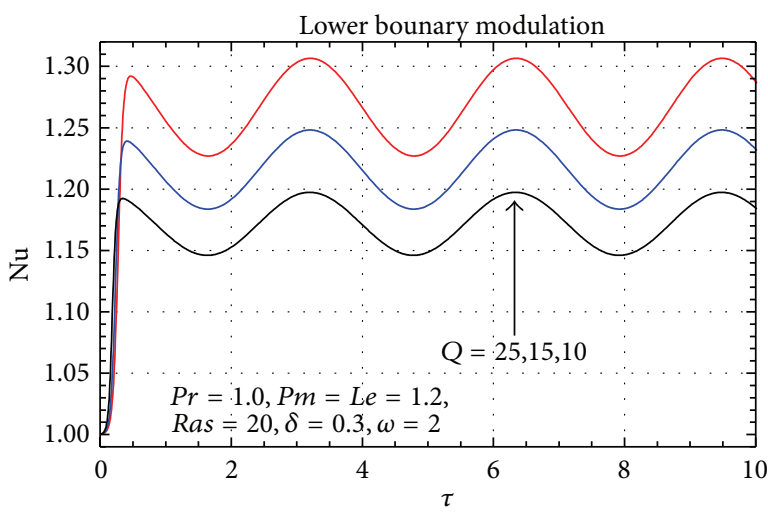

(a)

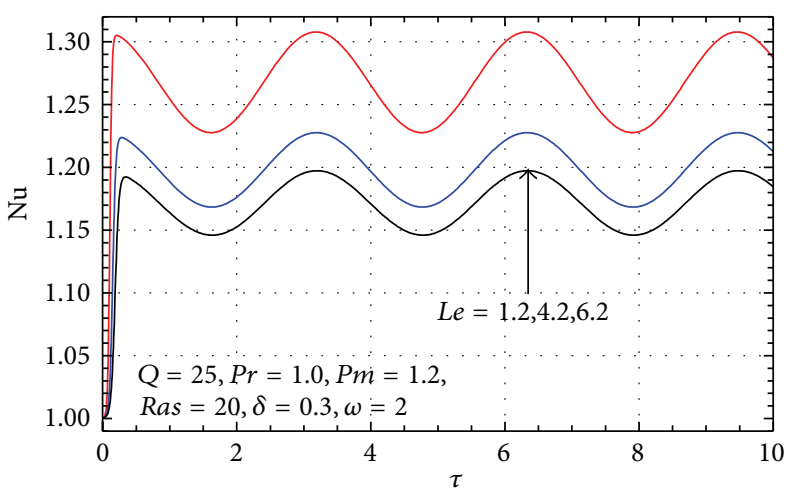

(c)

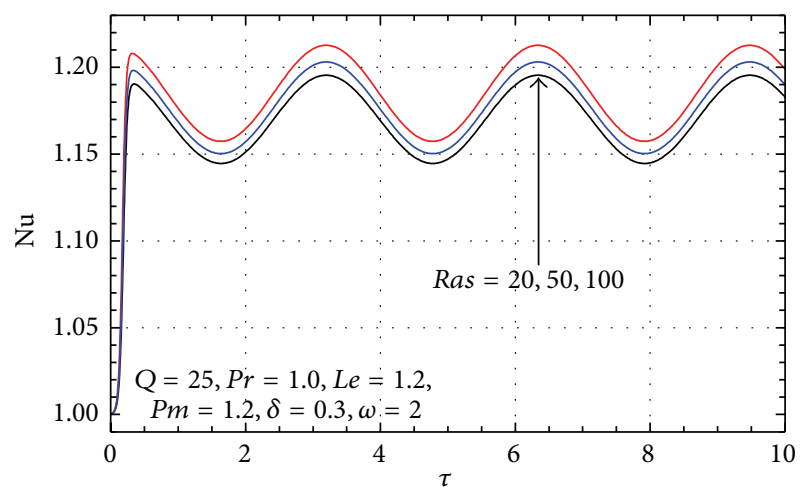

(e)

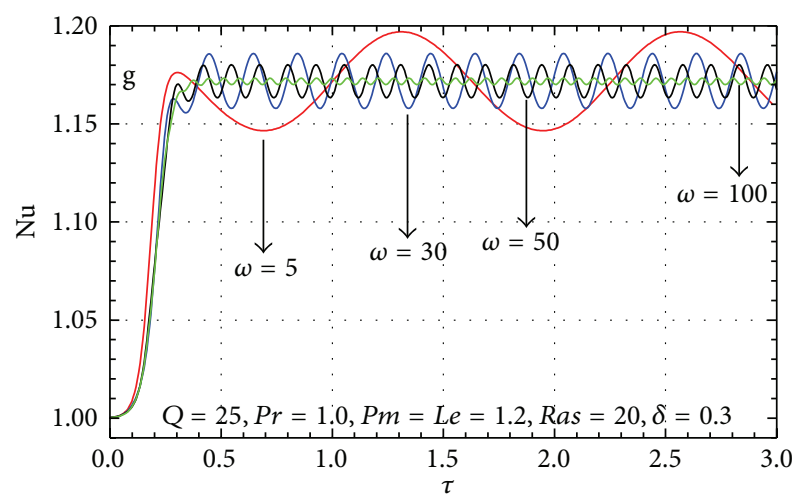

(g)

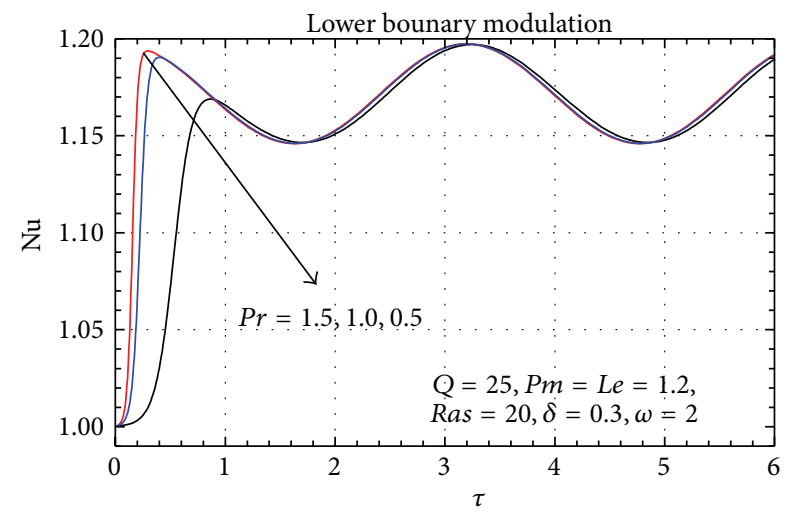

(b)

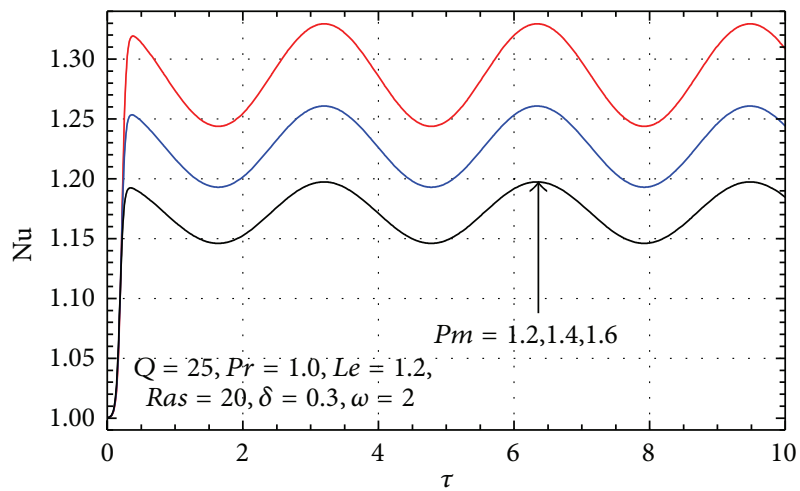

(d)

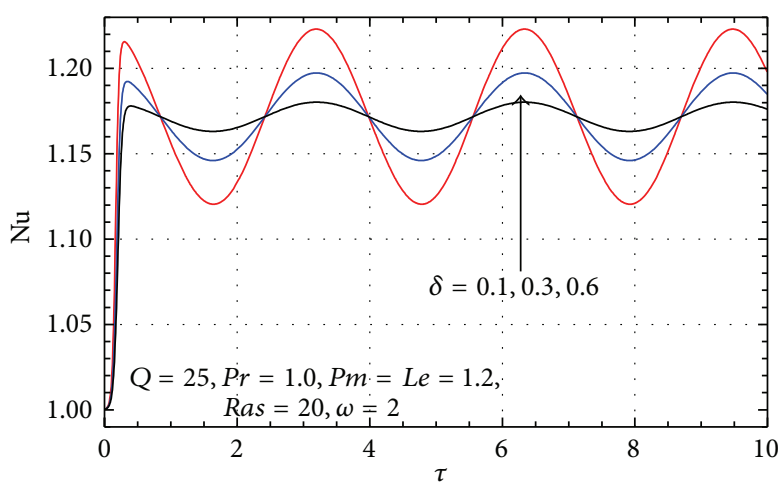

(f)

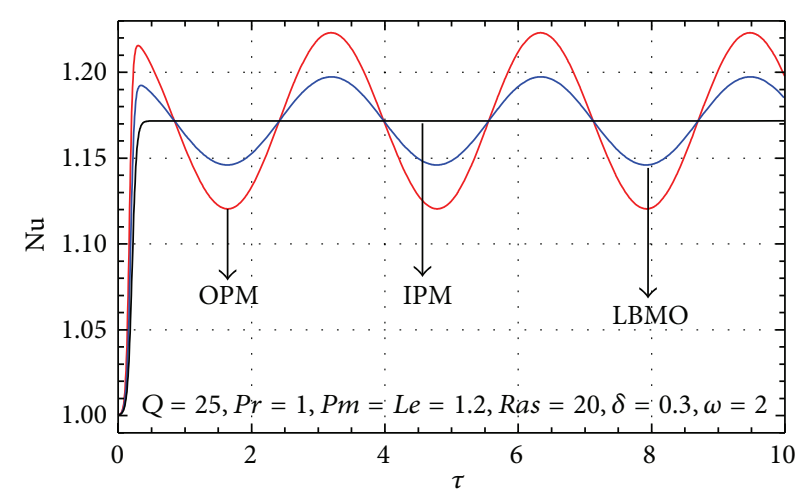

(h)

Figure 4: Nu versus $\tau$ (a) Q (b) $\operatorname{Pr}$ (c) Le (d) $\operatorname{Pm}$ (e) Ras (f) $\delta$ (g) $\omega$. 


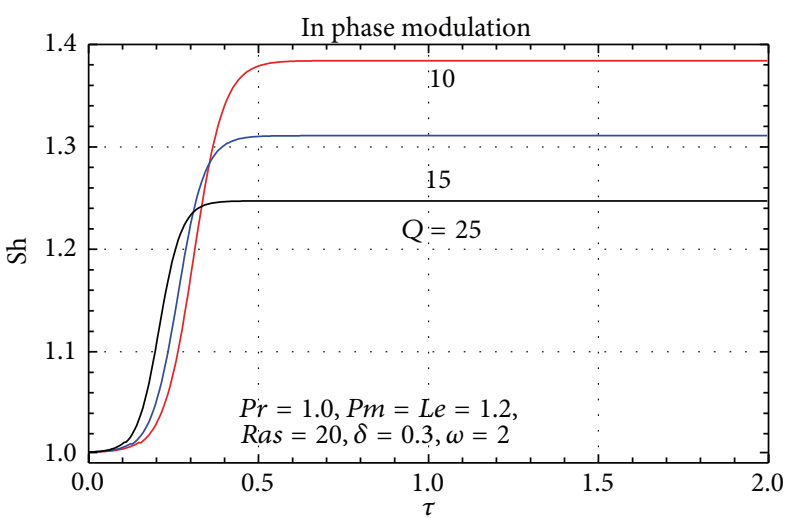

(a)

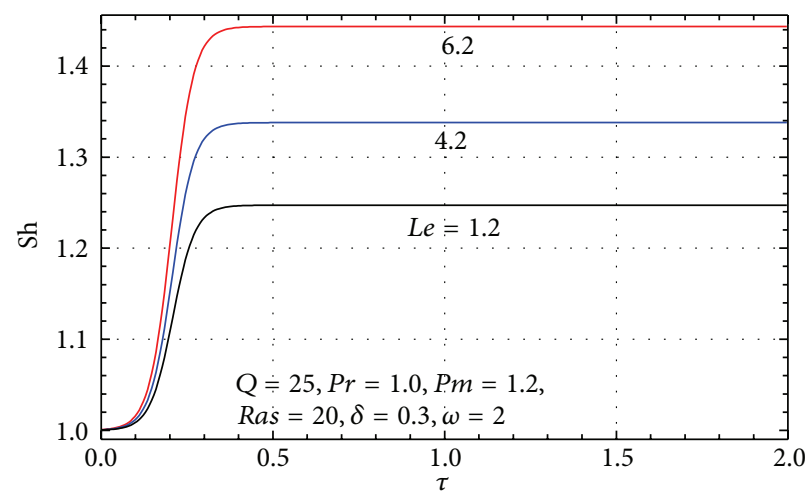

(c)

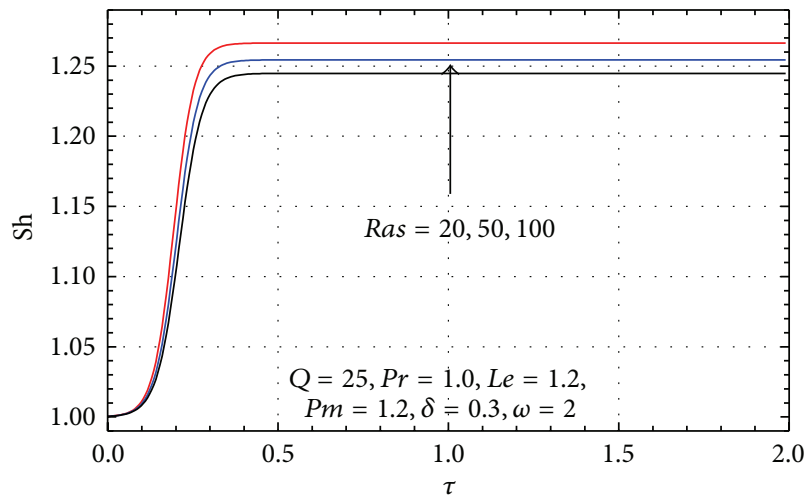

(e)

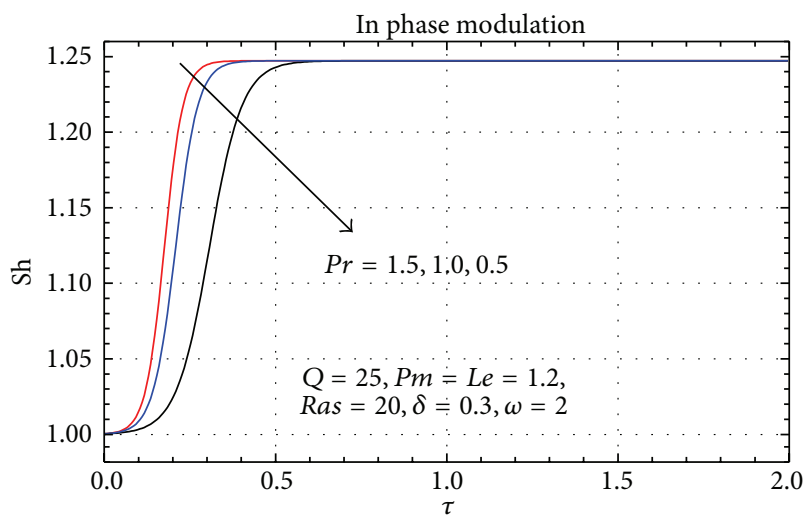

(b)

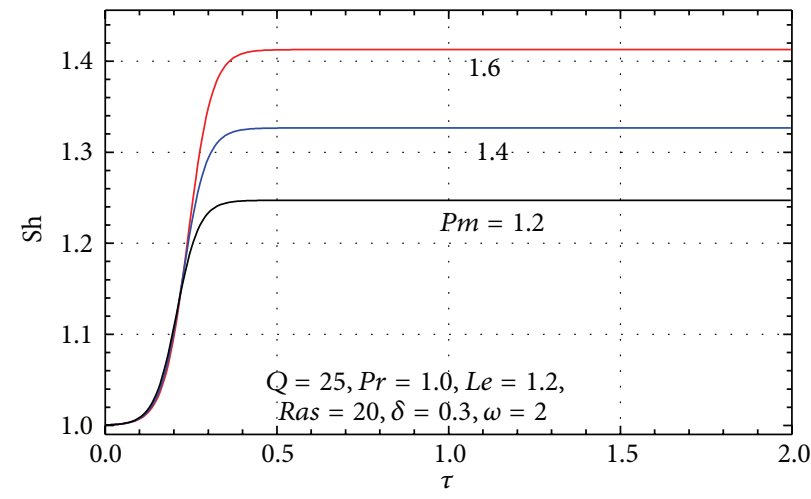

(d)

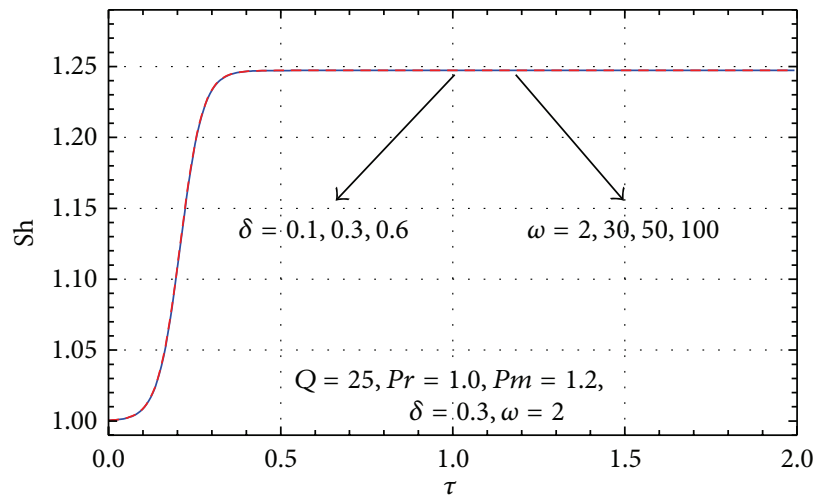

(f)

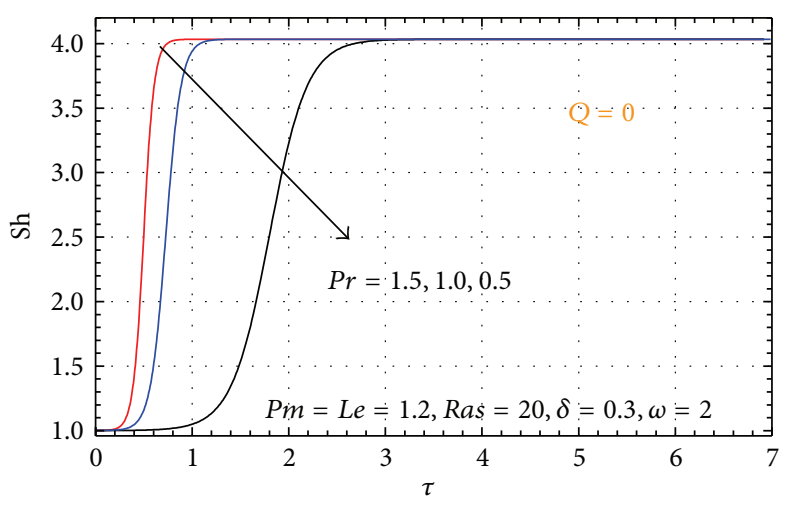

(g)

Figure 5: Nu versus $\tau$ (a) $Q$ (b) $\operatorname{Pr}$ (c) Le (d) $\operatorname{Pm}$ (e) $\operatorname{Ras}$ (f) $\delta, \omega$. 


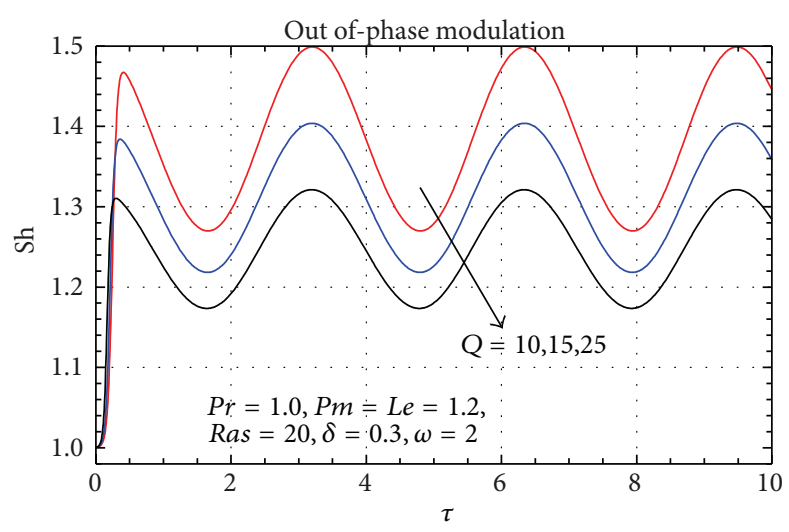

(a)

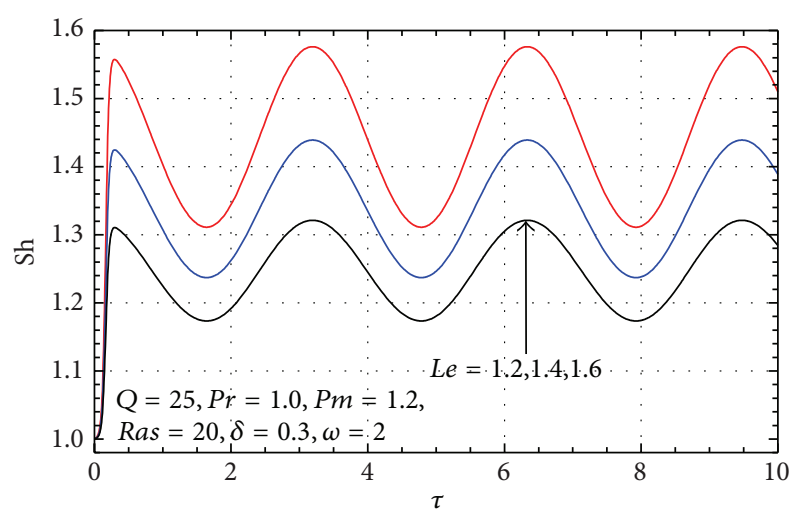

(c)

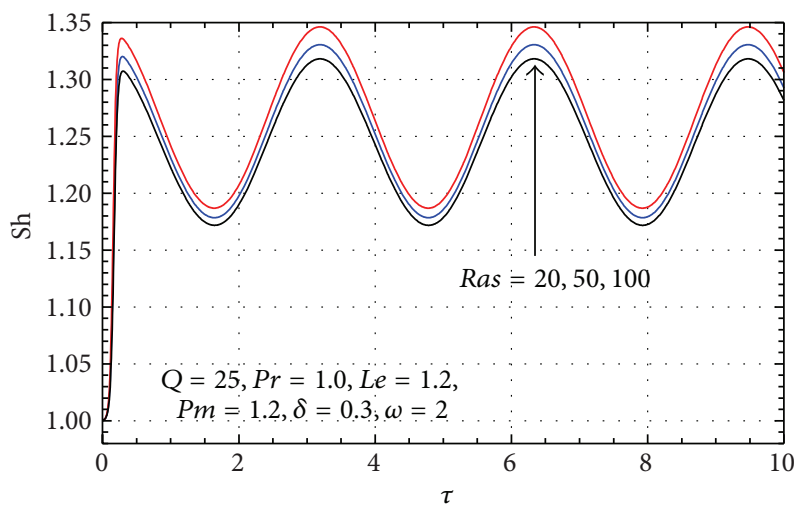

(e)

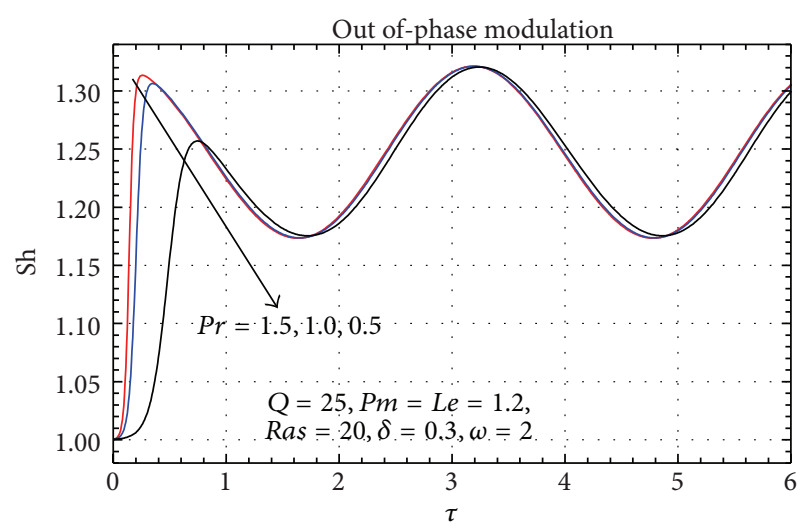

(b)

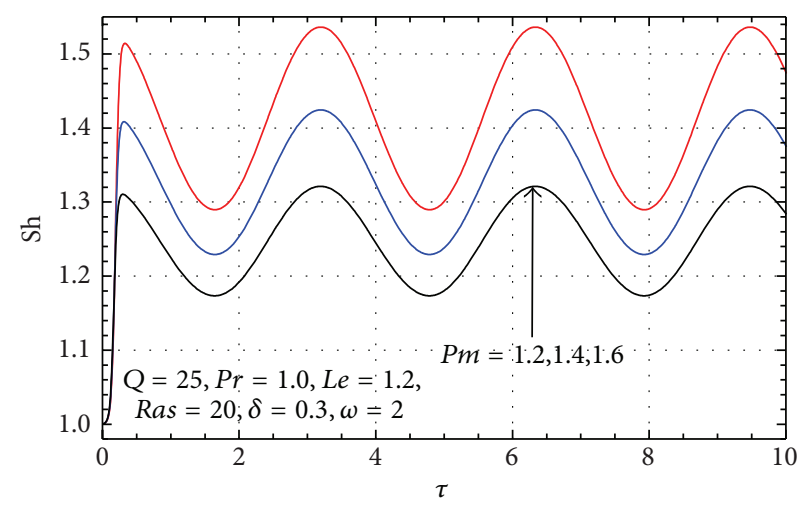

(d)

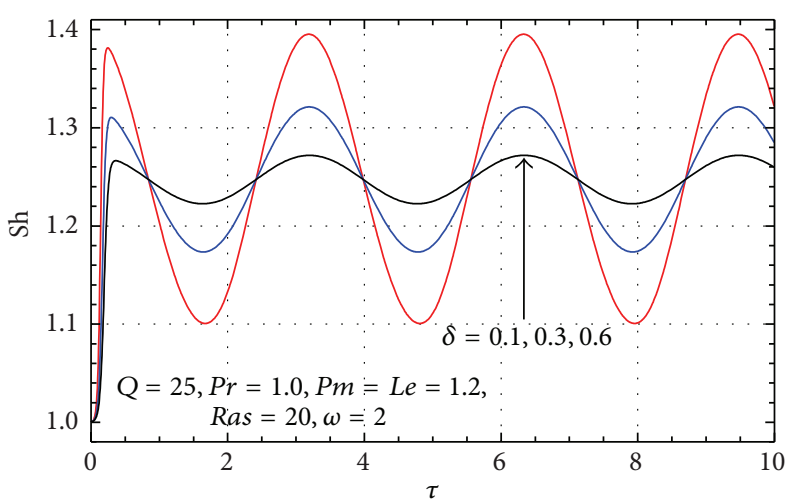

(f)

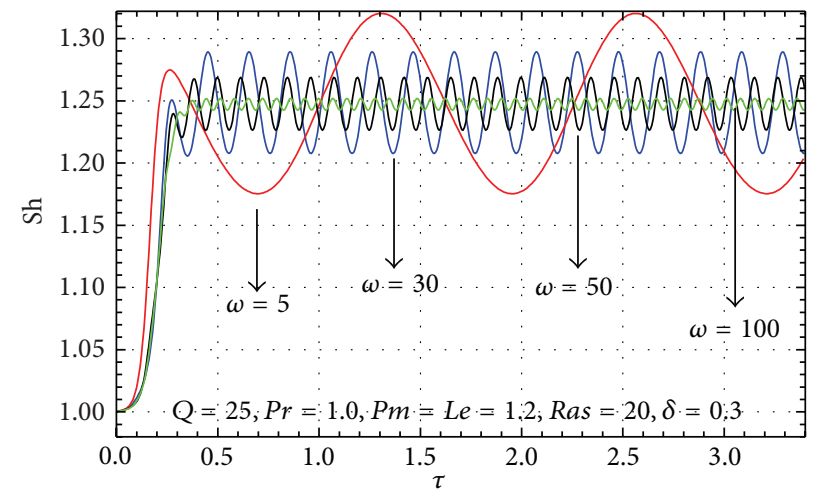

(g)

Figure 6: Sh versus $\tau$ (a) $Q$ (b) $\operatorname{Pr}$ (c) Le (d) $\operatorname{Pm}$ (e) Ras (f) $\delta$ (g) $\omega$. 


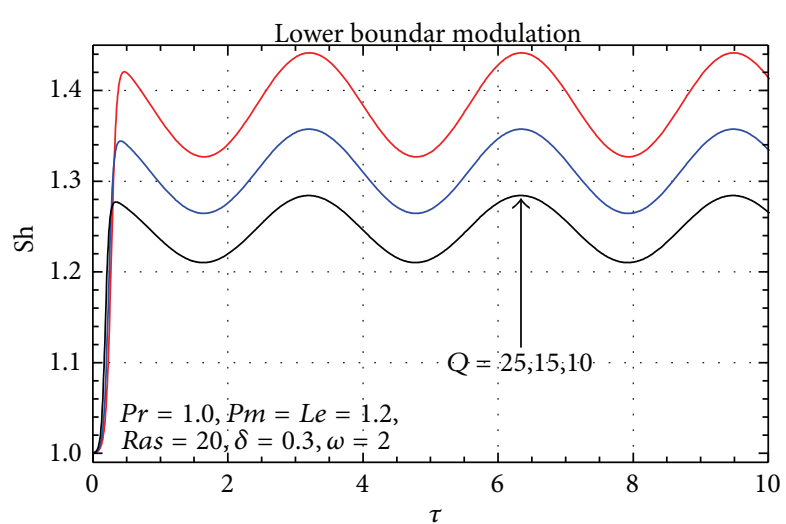

(a)

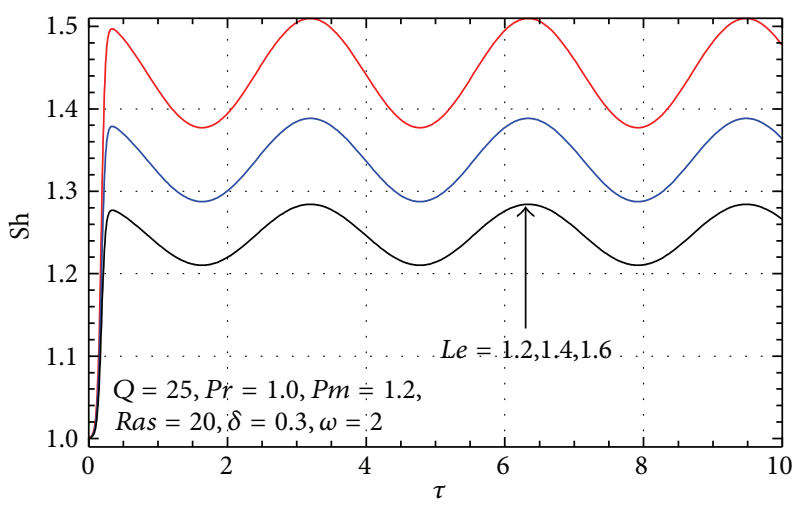

(c)

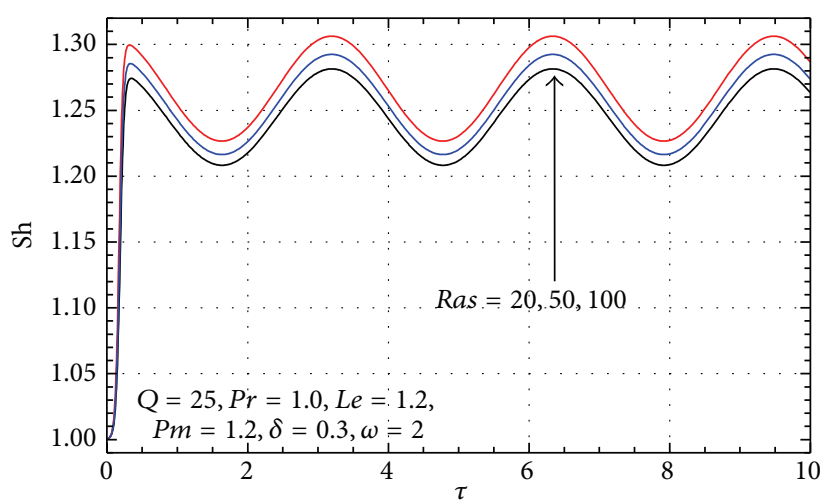

(e)

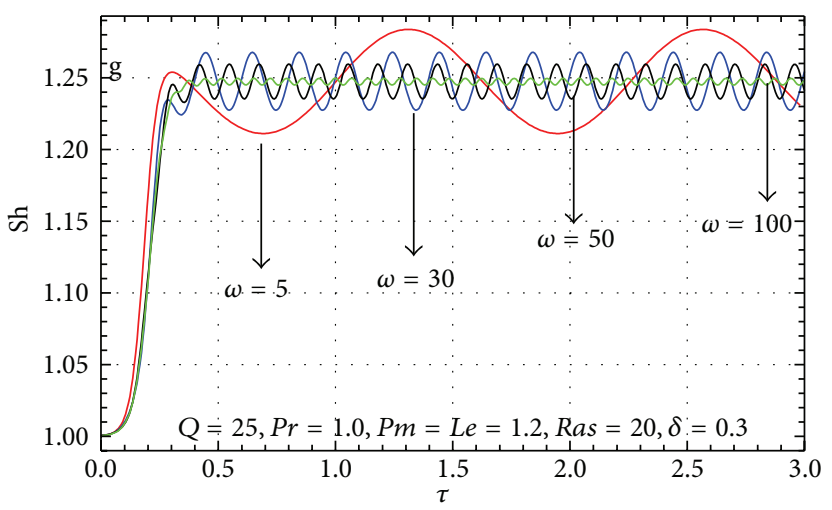

(g)

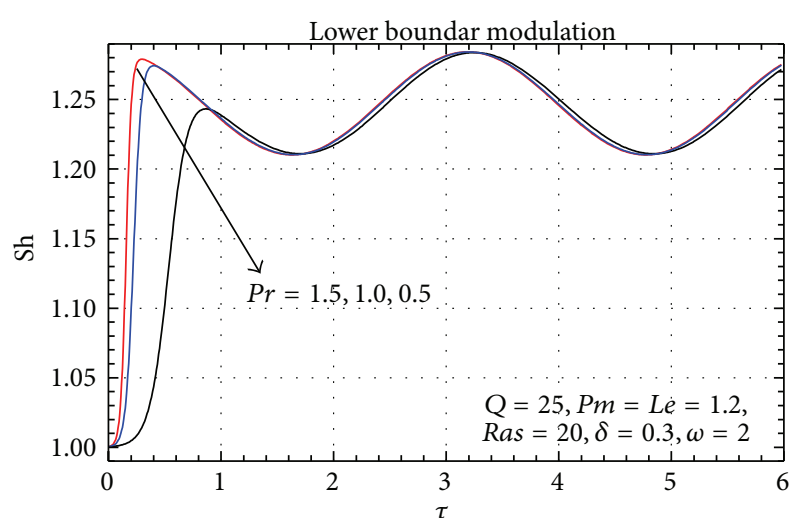

(b)

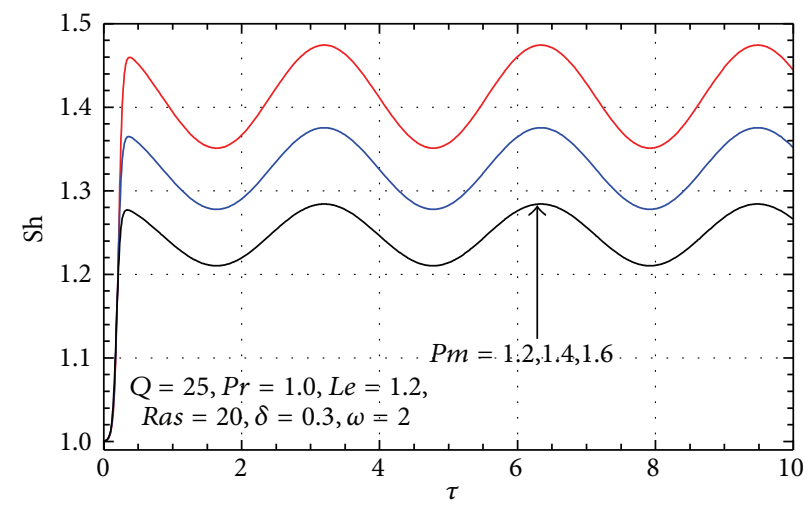

(d)

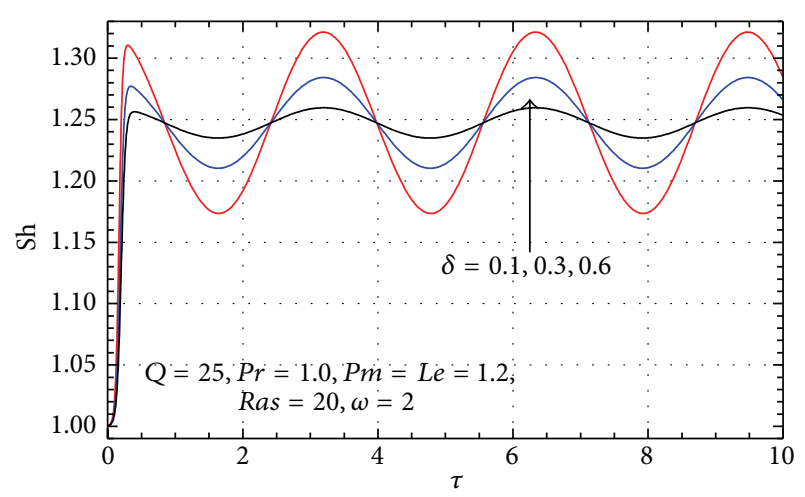

(f)

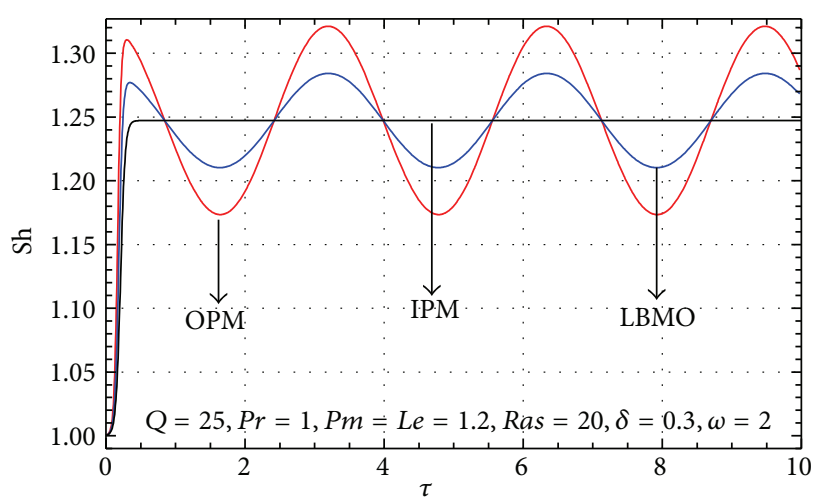

(h)

Figure 7: Sh versus $\tau$ (a) Q (b) $\operatorname{Pr}$ (c) Le (d) Pm (e) Ras (f) $\delta$ (g) $\omega$. 
(6) $[\mathrm{Nu} / \mathrm{Sh}]_{\text {Ras }=20}<[\mathrm{Nu} / \mathrm{Sh}]_{\text {Ras=50 }}<[\mathrm{Nu} / \mathrm{Sh}]_{\text {Ras=100 }}$, Figures 2(e)-7(e).

(7) $[\mathrm{Nu} / \mathrm{Sh}]_{\delta=0.1}<[\mathrm{Nu} / \mathrm{Sh}]_{\delta=0.3}<[\mathrm{Nu} / \mathrm{Sh}]_{\delta=0.5}$, Figures $3(\mathrm{f}), 4(\mathrm{f}), 6(\mathrm{f})$, and $7(\mathrm{f})$.

(8) $[\mathrm{Nu} / \mathrm{Sh}]_{\omega=100}<[\mathrm{Nu} / \mathrm{Sh}]_{\omega=50}<[\mathrm{Nu} / \mathrm{Sh}]_{\omega=30}<$ $[\mathrm{Nu} / \mathrm{Sh}]_{\omega=5}$, Figures $3(\mathrm{~g}), 4(\mathrm{~g}), 6(\mathrm{~g})$, and $7(\mathrm{~g})$.

\section{Conclusions}

The effect of temperature modulation on weak nonlinear double-diffusive magnetoconvection has been analyzed by using the nonautonomous Ginzburg-Landau equation. The following conclusions are drawn from previous analysis.

(1) The effect of IPM is negligible on heat and mass transport in the system.

(2) In the case of IPM, the effect of $\delta$ and $\omega$ is also found to be negligible on heat and mass transport.

(3) In the case of IPM, the values of $\mathrm{Nu}$ and Sh increase steadily for small values of time $\tau$; however $\mathrm{Nu}$ and $\mathrm{Sh}$ become constant when $\tau$ is large.

(4) The effect of increasing Pr, Le, Pm, Ras is found to increase in $\mathrm{Nu}$ and $\mathrm{Sh}$ thus increasing heat and mass transfer for all three types of modulations.

(5) The effect of increasing $\delta$ is to increase the value of $\mathrm{Nu}$ and Sh for the case of OPM and LBMO, hence heat and mass transfer.

(6) The effect of increasing $\omega$ is to decrease the value of $\mathrm{Nu}$ and $\mathrm{Sh}$ for the case of OPM and LBMO, hence heat and mass transfer.

(7) In the cases of OPM and LBMO, the natures of $\mathrm{Nu}$ and Sh remain oscillatory.

(8) Initially when $\tau$ is small, the values of Nusselt and Sherwood numbers start with 1 , corresponding to the conduction state. However as $\tau$ increases, $\mathrm{Nu}$ and $\mathrm{Sh}$ also increase, thus increasing the heat and mass transfer.

(9) The values of $\mathrm{Nu}$ and $\mathrm{Sh}$ for LBMO are greater than those in IPM but smaller than those in OPM.

(10) The effect of magnetic field is to stabilize the system.

\section{Nomenclature}

Latin Symbols

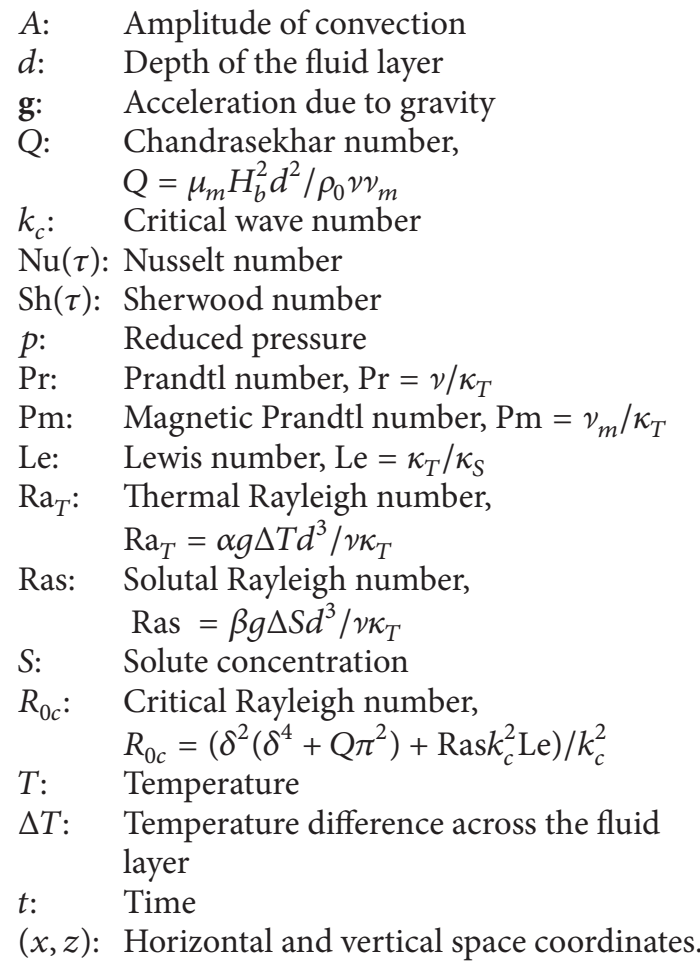

Greek Symbols

$\alpha: \quad$ Coefficient of thermal expansion

$\beta$ : Coefficient of solute expansion

$\delta^{2}$ : Horizontal wave number $\delta^{2}=k_{c}^{2}+\pi^{2}$

$\epsilon$ : Perturbation parameter

$\kappa_{T}$ : Thermal diffusivity

$\kappa_{S}$ : Solutal diffusivity

$\gamma:$ Heat capacity ratio $\gamma=(\rho c)_{m} /(\rho c)_{f}$

$\omega$ : Frequency of temperature modulation

$\delta: \quad$ Amplitude of temperature modulation

$\mu$ : Dynamic coefficient of viscosity of the fluid

$\mu_{m}$ : Magnetic permeability

$v$ : Kinematic viscosity, $v=\mu / \rho_{0}$

$v_{m}:$ Magnetic viscosity

$\rho: \quad$ Fluid density

$\psi:$ Stream function

$\psi^{*}$ : Dimensionless stream function

$\Phi:$ Magnetic potential

$\Phi^{*}$ : Dimensionless magnetic potential

$\tau$ : $\quad$ Slow time $\tau=\epsilon^{2} t$

$\theta: \quad$ Phase angle

$T^{\prime}$ : Perturbed temperature.

Other Symbols

$\nabla^{2}:\left(\partial^{2} / \partial x^{2}\right)+\left(\partial^{2} / \partial y^{2}\right)+\left(\partial^{2} / \partial z^{2}\right)$ 


\section{Subscripts}

$b$ : Basic state

$c$ : Critical

0 : Reference value.

\section{Superscripts}

I: Perturbed quantity

*: Dimensionless quantity.

\section{Conflict of Interests}

The authors declare that there is no conflict of interests regarding the publication of this paper.

\section{Acknowledgments}

This work was done during the lien sanctioned to the author by Banaras Hindu University, Varanasi, to work as professor of Mathematics at Department of Applied Mathematics, School for Physical Sciences, Babasaheb Bhimrao Ambedkar Central University, Lucknow, India. The author B.S. Bhadauria gratefully acknowledges Banaras Hindu University, Varanasi, for the same. Further, the author Palle Kiran gratefully acknowledges the financial assistance from Babasaheb Bhimrao Ambedkar Central University as a research fellowship.

\section{References}

[1] A. Akbarzadeh and P. Manins, "Convective layers generated by side walls in solar ponds," Solar Energy, vol. 41, no. 6, pp. 521$529,1988$.

[2] H. E. Huppert and R. S. J. Sparks, "Double-diffusive convection due to crystallization in magmas," Annual Review of Earth and Planetary Sciences, vol. 12, pp. 11-37, 1984.

[3] H. J. S. Fernando and A. Brandt, "Recent advances in double diffusive convection," Applied Mechanics Reviews, vol. 47, pp. c1c7, 1994 .

[4] J. S. Turner, Buoyancy Effects in Fluids, University Of Cambridge, 1979.

[5] N. Rudraiah and I. S. Shivakumara, "Double-diffusive convection with an imposed magnetic field," International Journal of Heat and Mass Transfer, vol. 27, no. 10, pp. 1825-1836, 1984.

[6] J. H. Thomas and N. O. Weiss, "The theory of sunspots," in Sunspots: Theory and Observations, p. 428, Kluwer Academic Publishers, Dordrecht, The Netherlands, 1992.

[7] W. B. Thompson, "Thermal convection in a magnetic field," Philosophical Magazine, vol. 42, pp. 1417-1432, 1951.

[8] S. Chandrasekhar, Hydrodynamic and Hydromagnetic Stability, Oxford University Press, London, UK, 1961.

[9] D. Lortz, "A stability criterion for steady finite amplitude convection with an external magnetic field," Journal of Fluid Mechanics, vol. 23, pp. 113-128, 1965.

[10] W. V. R. Malkus and G. Veronis, "Finite amplitude cellular convection," Journal of Fluid Mechanics, vol. 4, pp. 225-260, 1958.
[11] H. Stommel, A. B. Arons, and D. Blanchard, "An oceanographical curiosity: the perpetual salt fountain," Deep Sea Research, vol. 3, no. 2, pp. 152-153, 1956.

[12] K. Gotoh and M. Yamada, "Thermal convection of a horizontal layer of magnetic fluids," Journal of the Physical Society of Japan, vol. 51, no. 9, pp. 3042-3048, 1982.

[13] G. M. Oreper and J. Szekely, "The effect of an externally imposed magnetic field on buoyancy driven flow in a rectangular cavity," Journal of Crystal Growth, vol. 64, no. 3, pp. 505-515, 1983.

[14] N. Rudraiah, "Double-diffusive magnetoconvection," Pramana, vol. 27, no. 1-2, pp. 233-266, 1986.

[15] P. G. Siddheshwar and S. Pranesh, "Magnetoconvection in fluids with suspended particles under $1 \mathrm{~g}$ and $\mu \mathrm{g}$," Aerospace Science and Technology, vol. 6, no. 2, pp. 105-114, 2002.

[16] B. S. Bhadauria, "Combined effect of temperature modulation and magnetic field on the onset of convection in an electrically conducting-fluid-saturated porous medium," Journal of Heat Transfer, vol. 130, no. 5, Article ID 052601, 2008.

[17] P. G. Siddheshwar, B. S. Bhadauria, P. Mishra, and A. K. Srivastava, "Study of heat transport by stationary magneto-convection in a Newtonian liquid under temperature or gravity modulation using Ginzburg-Landau model," International Journal of NonLinear Mechanics, vol. 47, no. 5, pp. 418-425, 2012.

[18] P. G. Drazin and W. H. Reid, Hydrodynamic Stability, Cambridge University Press, Cambridge, UK, 2004.

[19] G. Venezian, "Effect of modulation on the onset of thermal convection," Journal of Fluid Mechanics, vol. 35, no. 2, pp. $243-$ 254, 1969.

[20] S. Rosenblat and G. A. Tanaka, "Modulation of thermal convection instability," Physics of Fluids, vol. 14, no. 7, pp. 1319-1322, 1971.

[21] M. N. Roppo, S. H. Davis, and S. Rosenblat, "Bénard convection with time-periodic heating," The Physics of Fluids, vol. 27, no. 4, pp. 796-803, 1984.

[22] B. S. Bhadauria and P. K. Bhatia, "Time-periodic heating of Rayleigh-Bénard convection," Physica Scripta, vol. 66, no. 1, pp. 59-65, 2002.

[23] B. S. Bhadauria, "Time-periodic heating of Rayleigh-Benard convection in a vertical magnetic field," Physica Scripta, vol. 73, no. 3, pp. 296-302, 2006.

[24] M. S. Malashetty and M. Swamy, "Effect of thermal modulation on the onset of convection in a rotating fluid layer," International Journal of Heat and Mass Transfer, vol. 51, no. 11-12, pp. 28142823, 2008.

[25] B. S. Bhadauria and P. Kiran, "Heat transport in an anisotropic porous medium saturated with variable viscosity liquid under temperature modulation," Transport in Porous Media, vol. 100, no. 2, pp. 279-295, 2013. 


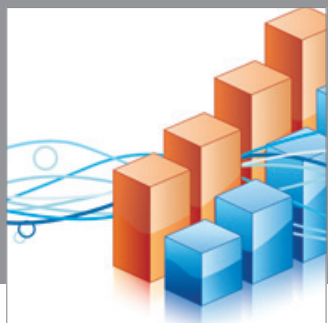

Advances in

Operations Research

mansans

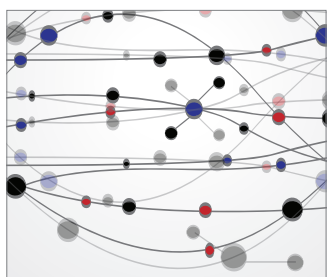

The Scientific World Journal
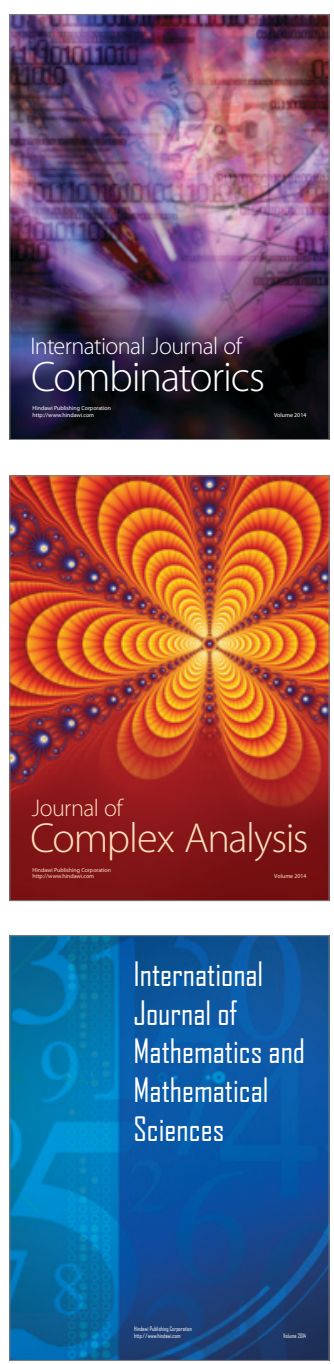
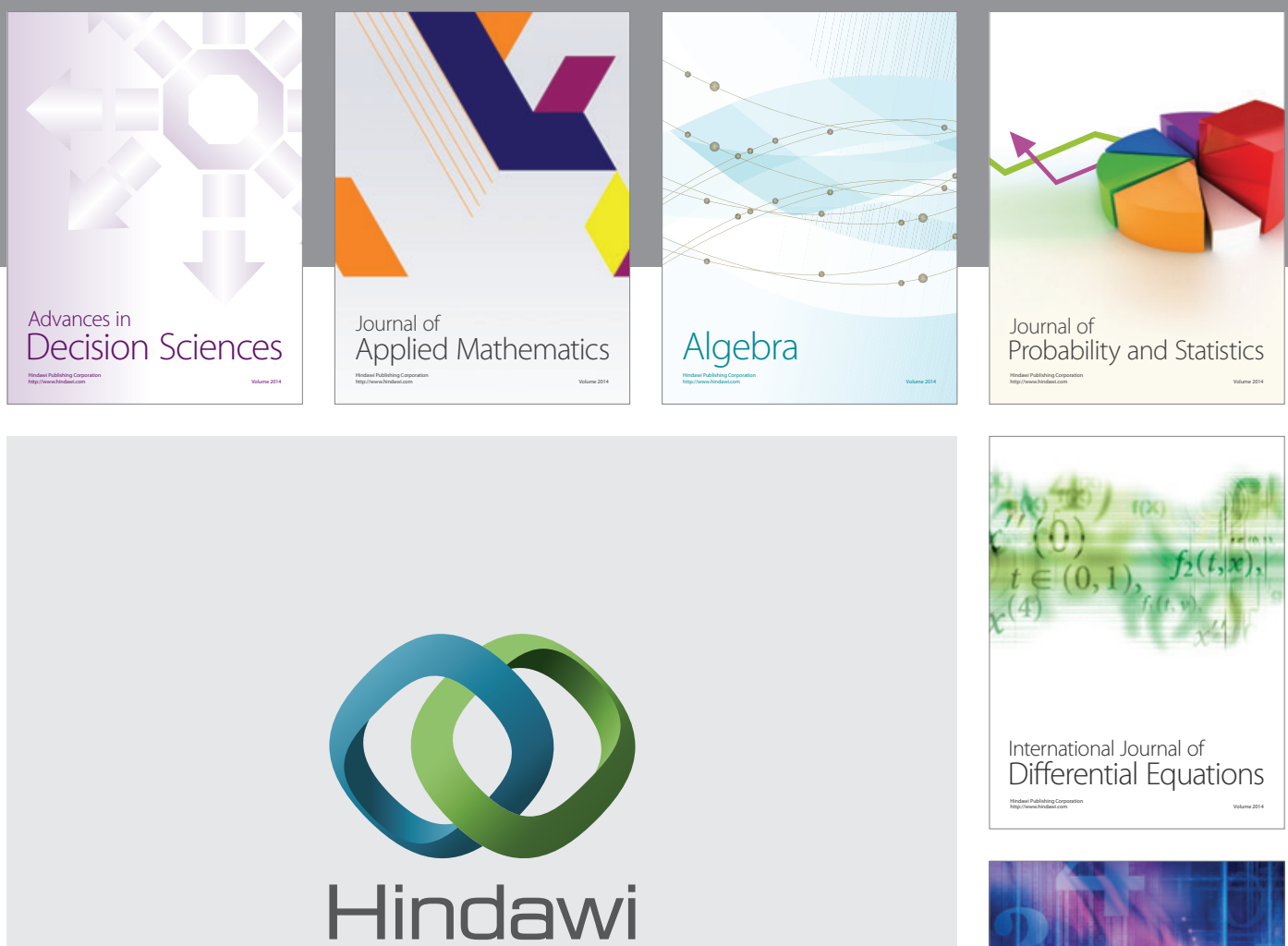

Submit your manuscripts at http://www.hindawi.com
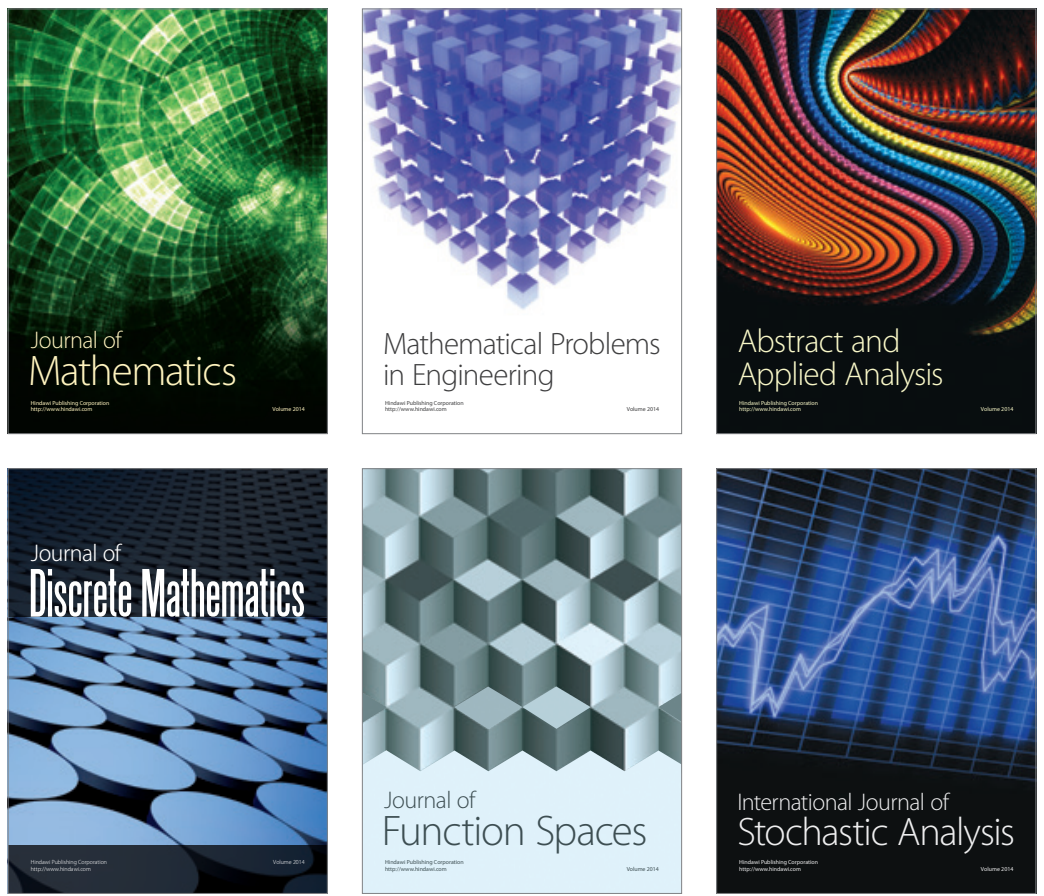

Journal of

Function Spaces

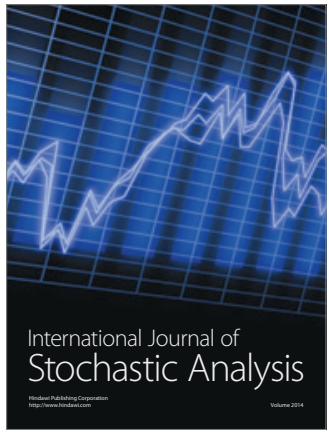

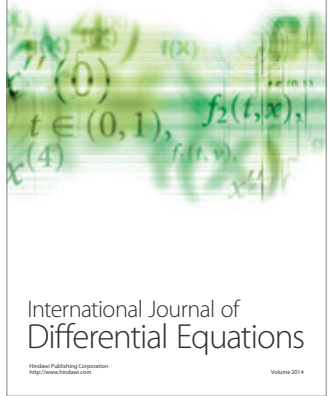
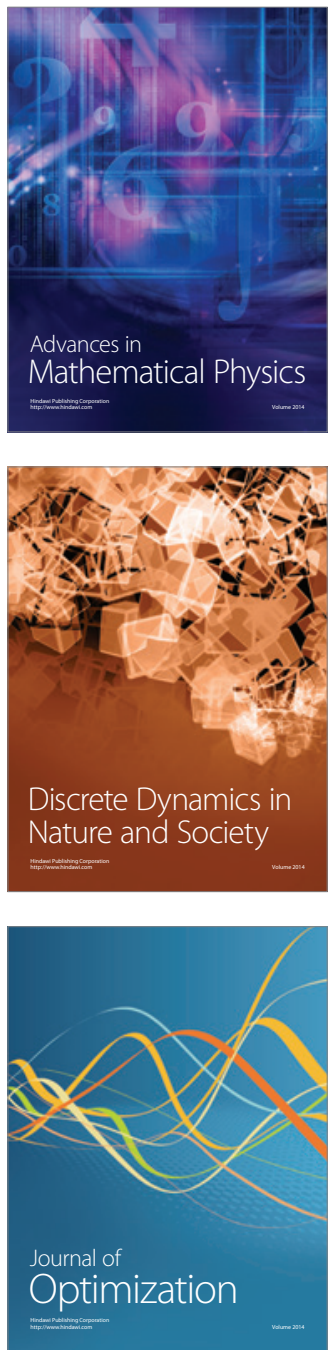